\title{
Ag-NYLON NANOCOMPOSITES BY DYNAMIC EMULSION POLYCONDENSATION
}

\author{
By \\ Linqi Zhang \\ Bachelor of Science in Mechanical Engineering \\ Harbin Institute of Technology \\ Harbin, Heilongjiang, China
}

2012

Submitted to the Faculty of the

Graduate College of the

Oklahoma State University

in partial fulfillment of

the requirements for the Degree of

MASTER OF SCIENCE

December, 2014 


\section{Ag-NYLON NANOCOMPOSITES BY DYNAMIC EMULSION POLYCONDENSATION}

Thesis Approved:

Dr. A. Kaan Kalkan

\begin{tabular}{c} 
Associate professor of Mechanical and Aerospace Engineering \\
Thesis Adviser \\
Dr. Nicholas F. Materer \\
\hline Associate professor of Chemistry \\
Committee Member \\
Dr. Sandip P. Harimkar \\
\hline Associate professor of Mechanical and Aerospace Engineering \\
Committee Member
\end{tabular}




\section{ACKNOWLEDGEMENTS}

First and foremost, I would like to express my acknowledgement to my adviser, Dr. A. Kaan Kalkan, for his encouragement, patience, and the exciting project he offered. Without his support, this thesis would not have been possible.

I would also like to thank Dr. Sandip P. Harimkar and Dr. Nicholas F. Materer for providing us the laboratory resources. I also thank Dr. Jacobs of Undergraduate Teaching Laboratory (UTL) in Chemistry Department for his training on DSC.

I am really thankful to my colleague, Sriharsha, who has been a great help, especially with Raman spectroscopy, FTIR, SEM, nano-indentation and silver nanowire synthesis. I also thank my colleague, Özge, for her assistance in ordering the chemicals and supplies. Additionally, I cannot disregard the help of Salah and Habib in obtaining the nano-indentation and XRD data. I am also indebted to all my other colleagues in the Functional Nanomaterials Laboratory for their support and accompany.

Finally, I would like to thank my parents for their support and trust during my study. 
Name: LINQI ZHANG

Date of Degree: DECEMBER, 2014

Title of Study: Ag-NYLON NANOCOMPOSITES BY DYNAMIC EMULSION

POLYCONDENSATION

\section{Major Field: MECHANICAL AND AEROSPACE ENGINEERING}

Abstract: Thermoplastics (plastics) have revolutionized human life due to their low cost and ease of fabrication. A common and effective approach to mechanical enforcement of thermoplastics is inclusion of micro- or nanofillers of higher strength and elastic modulus. Conventionally, the fillers are dispersed by shear mixing in molten polymer, where viscosity of the mixture is dramatically increased due to large interface area. Accordingly, the dispersion is not efficient and filler content is typically limited to below $5 \%$ by weight. The present thesis work develops a novel technique for dispersing nanofillers in a thermoplastic polymer where polycondensation and dispersion of the nanofillers occur simultaneously via dynamic emulsion polycondensation at ambient temperature. The composite is manufactured in the form of a uniform powder, which can be molded into desired shape by melting.

The technique is demonstrated for silver nanowire - Nylon 66 nanocomposites. In this demonstration, silver nanowires are synthesized by polyol process. Polyvinylprrolidone (PVP) is used to functionalize the silver nanowires. Composites with silver weight fraction of $1.49 \%, 3.28 \%, 6.74 \%$ are prepared and characterized by SEM, TEM, UV-Vis, Raman, FTIR, DSC and nano-indentation. The silver nanowires are found to be monodispersed and hydrogen-bonded to the Nylon 66 matrix through PVP. Glass transition temperature of the composites decreases from 61 to $48{ }^{\circ} \mathrm{C}$ with silver weight fraction increasing from 0 to $6.47 \%$. The decrease of the glass transition temperature is owed to the plasticizer effect as well as heterogenous nucleation effect of the nanowires for polymerization leading to shorter chain length. Finally, mechanical properties of the composites show insignificant variation with the filler content. This absence of the composite effect is explained by Halpin-Tsai model, where the filler enforcement and decrease in matrix modulus counterbalance. 


\section{TABLE OF CONTENTS}

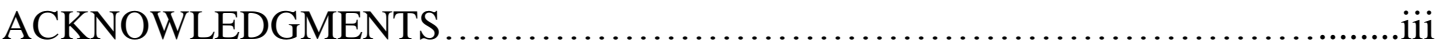

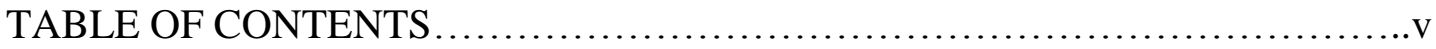

LIST OF FIGURES ...........................................................

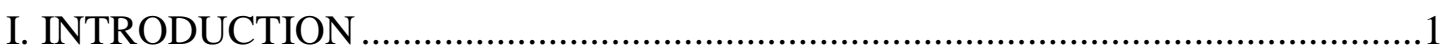

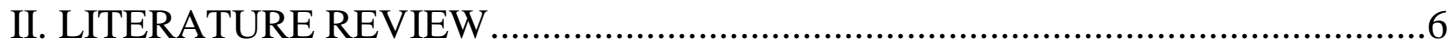

2.1. Studies of nylon composites .....................................................................

2.1.1. Studies on mechanical properties of the nylon composites ......................6

2.1.2. Studies on crystallization behavior of the nylon composites....................8

2.1.3. Studies on performance of the nylon composites ...................................10

2.2. Localized surface plasmon resonance of silver nanowires .............................12

2.3. Polyol synthesis of AgNWs ...........................................................................13

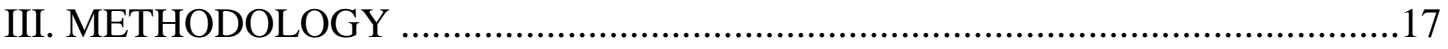

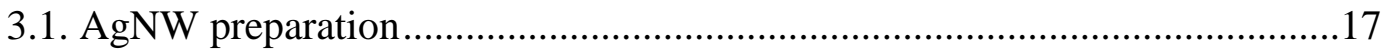

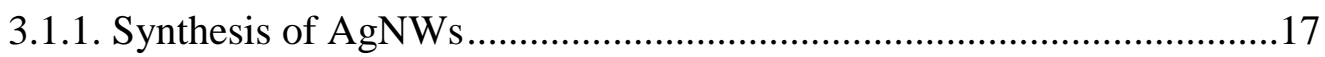

3.1.2. Separation and washing of AgNWs .......................................................19

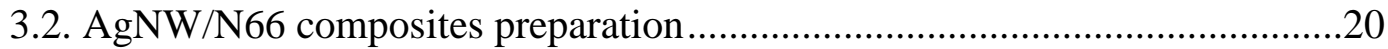

3.2.1. Conventional interfacial polycondensation of N66 ..............................20

3.2.2. AgNW/N66 composite synthesis .......................................................21

3.2.3. Separation and cleaning of AgNW/N66 microparticles ........................22

3.3. Theoretical calculation of silver weight fraction in N66 ..............................22

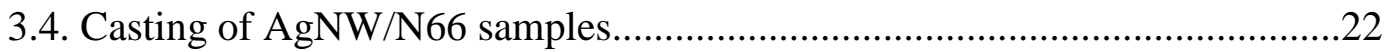

3.5. Characterization of the composites ...........................................................24 
3.5.1. UV-Vis spectroscopy

3.5.2. Scanning electron microscopy ....................................................24

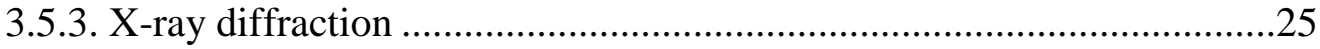

3.5.4. Raman spectroscopy ................................................................25

3.5.5. Fourier transform infrared spectroscopy ......................................25

3.5.6. Differential scanning calorimeter ..............................................26

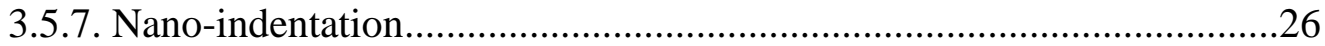

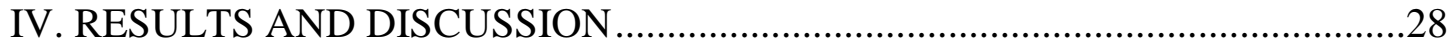

4.1. Structural characterization of AgNWs .................................................28

4.2. Physical characterization of AgNW/N66 composite microparticles ..............32

4.3. Fourier Transform Infrared Spectroscopy ................................................. 34

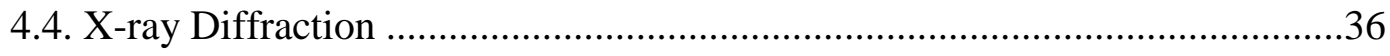

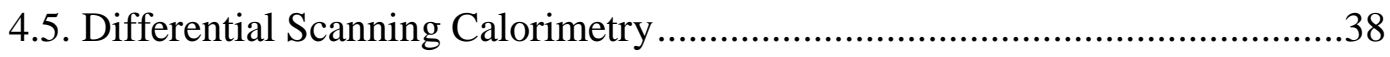

4.6. Mechanical properties by nano-indentation ................................................41

V. CONCLUSION AND FUTURE WORK …....................................................45

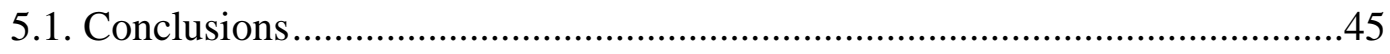

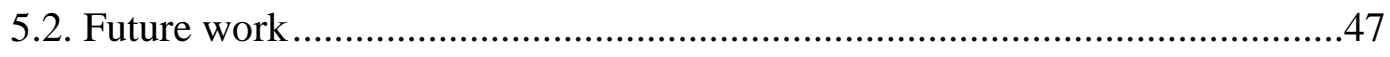

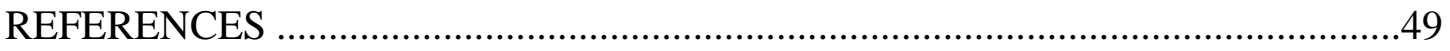




\section{LIST OF FIGURES}

Figure 2.1: Schematic illustrating the N66 structure ...............................................

Figure 2.2: Schematic illustration of super-cooling ..............................................10

Figure 2.3: Schematic illustration of LSPR transverse electron oscillation for AgNWs

Figure 2.4: a) Schematics illustrating growth mechanisms of different $\mathrm{Ag}$ nanostructures. b) Schematic illustration of the growing mechanism of AgNWs. ...14

Figure 3.1: Photograph of the experimental setup for the synthesis of AgNWs .......18

Figure 3.2: Schematic illustration of interfacial polycondensation ..........................20

Figure 3.3: Schematic illustration of AgNW/N66 composite synthesis and separation

Figure 3.4: a) Photograph illustrating the sample casting. b) Glass vial aluminum cap

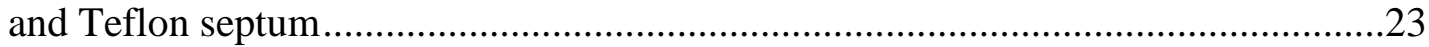

Figure 3.5: AgNW/N66 samples prepared for nano-indentation................................24

Figure 3.6: Graphical illustration of a Raman acquisition.........................................26

Figure 4.1: Average optical extinction spectrum of the AgNWs after 8 batches with error bars

Figure 4.2: SEM image of the AgNWs synthesized by the polyol process

Figure 4.3: a) Raman spectrum of PVP. b) Raman spectrum of AgNWs (functionlized by PVP)

Figure 4.4: AgNW/N66 composite microparticles. ...................................................32

Figure 4.5: Optical microscope image of AgNW/N66 microparticles .......................33

Figure 4.6: SEM image of AgNW/N66 composite microparticles..............................33

Figure 4.7: FTIR spectra of the commercial N66 and N66 in this work ....................35

Figure 4.8: FTIR spectra of the PVP and the AgNW/N66 composite particles .........35 
Figure 4.9: XRD spectra of AgNW/N66 composite particles. The inset shows AgNW:N66 mass ratio versus AgNW:N66 XRD intensity ratio

Figure 4.10: a) Crystallization diagram for AgNW/N66 composites. b) Melting diagram for AgNW/N66 composites.

Figure 4.11: Schematic illustration of free volume formation in AgNW/N66 composite .40

Figure 4.12: Negative $\Delta \mathrm{T}_{\mathrm{g}}$ versus AgNW content.............................................40

Figure 4.13: Load-displacement curve of AgNW/N66 composites.........................43

Figure 4.14: Modulus and hardness of AgNW/N66 composite samples.....

Figure 4.15: Composite modulus for constant versus decreasing matrix modulus with

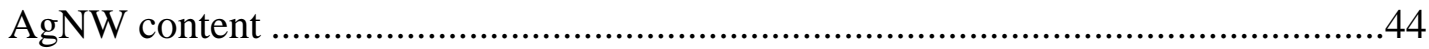




\section{CHAPTER 1}

\section{Introduction}

Thermoplastics (plastics) have revolutionized human life due to their low cost and ease of fabrication [1]. A thermoplastic can be molten or softened at relatively low temperatures and subsequently shaped to any shape. The downfall of thermoplastics is their poorer mechanical strength and stiffness [2]. This shortcoming is traditionally addressed by two approaches. The first is the crosslinking of polymer chains. The second is the composite approach, which involves inclusion of micro- or nanofillers of higher strength and elastic modulus in the plastic matrix. The fillers can also impart additional enhanced attributes to the composite such as fire-retardant $[3,4]$, diffusion-barrier [5, 6], antibacterial [7], anti-static [8] and thermal/electrical conduction [8-10] characteristics.

With the invention of nanostructured materials in recent years (e.g., carbon nanotubes, metal/ceramic nanowires, graphene), the size of fillers in thermoplastics has gradually transitioned from micron to nanometer scale [11]. In terms of mechanical properties, the major benefit gained at the nanoscale is higher interface area per volume that increases the total crosslinking between the filler and polymer matrix phases. Consequently, the shear stress at the interface can be reduced by orders of magnitude that prevents slip and allows better strain (i.e., also stress and load) transfer. Second, in brittle materials such as ceramics, mechanical failure (i.e., crack) may start from a single defect or stress concentration site and propagate over the whole body of the material. In nanocomposites, dividing the fillers to much smaller 
volumes decreases the number of defect and stress concentrator sites per filler (i.e., nanoparticle) and increases its survival rate under extreme loading. The nanometer size of the fillers also account for novel chemical, optical and other physical properties [12-14].

A major challenge in the production of nanocomposites is uniform dispersion of the nanofillers without aggregation (i.e., monodispersion). In case of excessive aggregation, the aggregates behave as larger particles and the advantages of the nanoscale are lost. Further, weak bonding between the aggregated fillers results in poor mechanical properties. Conventionally, the fillers are dispersed by shear mixing in either molten polymer or polymer dissolved in a solvent. In both cases, the viscosity of the mixture is dramatically increased due to large interface area between the polymer and fillers. Therefore, efficient dispersion is disabled. Increasing the temperature of the polymer melt lowers viscosity, but it also increases the tendency of the polymer to oxidize. Similarly, the polymer concentration in the solvent could be diluted to retain lower viscosity, but this occurs at the expense of increased burden of evaporating the solvent after the mixing process. Additionally, many common polymers are soluble in carcinogenic solvents and some have limited solubility. Thus, efficient dispersion of the nanofillers in polymer melts and solutions has consequences. Accordingly, the filler content is typically limited to below 5\% by weight [15].

The present thesis work has developed a different and novel technique for efficient and low-cost dispersion of nanofillers in a thermoplastic polymer. In this technique, polycondensation and dispersion of the nanofillers occur simultaneously. Because the polymer precursors are dissolved in a low viscosity solution, the nanofillers are efficiently dispersed (i.e., suspension) 
prior to polymerization. As the polymerization proceeds, the nanofillers get entrapped in the solid polymer, but they remain highly monodispersed.

In particular, the present thesis demonstrates a silver nanowire - Nylon 66 (AgNW/N66) composite using interfacial polycondensation. Interfacial polycondensation is a well-established polymerization technique which takes place at the interface between two mutually immiscible solvents. Typically, one is polar dissolving a polar monomer, the other is nonpolar dissolving a nonpolar monomer. The two monomers react at the interface accounting for the condensation polymerization reaction. In a typical process, it is known that polar monomer can partially dissolve the nonpolar solvent while the nonpolar monomer is essentially not soluble in the polar solvent. Therefore, the reaction is believed to occur effectively in the nonpolar solvent but at close proximity of the interface. The polymer product accumulating at the interface in the form of a thin film has to be simultaneously removed, which otherwise blocks the monomer diffusion to the interface from polar to nonpolar side [16].

One major novel aspect of the present thesis work is the incorporation of AgNWs to the polymer during polycondensation. In the present case, the AgNWs are synthesized by polyol process and capped with polyvinylpyrollidone (PVP), a polar polymer. It is shown that the AgNWs are efficiently dispersed in N66 at room temperature.

The second novelty in the developed technique is aggressive mixing of the two solvents by a vortexer during the reaction rather than keeping a static and flat interface between them. The aggressive mixing basically disperses the nonpolar solvent in the polar solvent in the form of microdroplets (i.e., emulsion) and hence dramatically increases the interface area between the two domains leading to a much faster polymerization rate. It is found that N66 polymerizes in the 
form of microspheres with AgNWs entrapped in them. Once the condensation reaction is complete, the AgNW-dispersed N66 microspheres are centrifuged and dried to microparticles, which are subsequently molten and cast into a mold to achieve high filler content bulk nanocomposite without the need for any shear mixing. Accordingly, we adopt the terminology: Nanocomposites by Dynamic Emulsion Polycondensation (NCDEP).

The present work has also modified the conventional interfacial polycondensation technique by using partially miscible solvents. Specifically, water and tetrahydrofuran (THF) are used. Partial solubility (albeit low) of the solvents allows a higher density of AgNWs transferred into N66 particles. It is inferred that the polycondensation occurs in microdroplets of THF, where the two monomers and AgNWs all come together.

Another unique attribute of the developed technology is the strong binding of AgNWs to the N66 matrix via ultrathin PVP capping around the AgNWs (i.e., coupling agent) as corroborated by vibrational spectroscopy in the present thesis. PVP strongly binds to Ag surface selectively in the [110] directions and thereby leads to directional growth of the AgNWs in the [111] direction during the polyol process. Further it establishes conformal hydrogen bonding with N66 and thereby serves as the coupling agent.

The organization of the present thesis is as follows. Chapter 2 reviews various properties and applications of N66. Additionally, the polyol synthesis of AgNWs and localized plasmon modes in AgNWs are reviewed. In Chapter 3, experimental procedures for AgNW synthesis and AgNW/N66 composite fabrication are detailed. Chapter 4 presents the material characterization results. In particular, SEM and UV-Vis are used to show the morphology and quality of AgNWs and composite microparticles. Raman and FTIR are used to corroborate the bonding between 
AgNWs, PVP and N66. DSC is conducted to investigate the thermal behavior of the composites. The variation of mass ratio between AgNWs and N66 is determined by XRD. Mechanical properties are studied by nano-indentation and explained in the view of the Halpin-Tsai model. Finally, various conclusions are drawn as well as future research directions are suggested in Chapter 5 . 


\section{CHAPTER 2}

\section{Literature Review}

\subsection{Studies on nylon composites}

Nylon was first synthesized by DuPont in 1938. Since then, it has become the most widely used thermoplastic. Nylon has been extensively utilized by automobile, electronic, chemical, medical and food industries due to its low cost, easy processing and good workability. However, among all the nylon products, pure nylon is rarely used due to three reasons. First, nylon (i.e., N66) shows strong polarity. It absorbs water in the ambient and therefore exhibits dimensional instability. Second, nylon has poor mechanical strength and stiffness. This shortcoming greatly limits its application as load/impact-bearing material. Third, nylon is sensitive to light, acids and oxidants. Therefore, development of modified nylon with improved mechanical and chemical properties and multi-functionalities has been a topic of interest for several decades.

\subsubsection{Studies on mechanical properties of the nylon composites}

Several groups have developed nylon composites to enhance the mechanical strength and modulus of pure nylon. Shiao et al. have investigated N66/glass-fiber composites. Their results showed that the elastic modulus of the composites increased from 2 to $6 \mathrm{GPa}$ when the glass fiber weight fraction is increase from 0 to $45 \%$ [17]. In addition to synthetic fibers like glass fibers, 
natural fibers can also be used to reinforce polymers. Tremolite is a non-metallic mineral with length-to-diameter ratio of 24:1. Baji et al. have reinforced N66 fiber with 7.5 wt\% CNT. Their results showed that at room temperature, the storage modulus of the nylon composite is $3.3 \mathrm{GPa}$ which is $250 \%$ higher than the unfilled one [18].

With the advent of nanotechnology, carbon nanotubes (CNTs) have found applications in polymer-based composites due to their excellent mechanical properties. Zeng at al. have investigated the Nylon 1010/Multi-walled CNTs (N1010/MWCNTs) composites. Tensile tests showed that the Young's modulus of N1010/MWCNT composites improved by $87.3 \%$ when MWCNTs weight fraction is $30 \%$. DMA results showed that the storage modulus of the composite increased by $197 \%$ (at $0{ }^{\circ} \mathrm{C}$ ) [19]. In another study, Kang et al. have studied N610/MWCNT composites. Tensile tests showed a 170\% increase in Young's modulus, 40\% increase in tensile strength and 35\% elongation at failure when MWCNT weight fraction was 1.5\% [20]. To the best of the Author's knowledge, the enforcement of N66 by AgNWs has not been studied yet.

Toughness is an important requirement for thermoplastic composites, especially when they are used as impact-bearing materials. Fillers, especially fibers in the nylon matrix, will greatly improve the impact resistance of the composites. First, the fibers in the matrix will stop the crack propagation and hold the matrix together. Second, fractures occurring by fiber pull-out or breakage consume more energy than the matrix breaking by itself. It has been observed by Akay et al. that crack initiation force and total energy fracture values were $25-37 \%$ and $300-600 \%$ larger in N66/glass fiber composite (50 wt\%) than those in pure N66, respectively [21]. Surfactants are proved to be excellent coupling agents in composites. Surfactants may provide 
copulations between the fillers and the matrices to improve their interface conditions. Furthermore, surfactants may impede the filler aggregation. Liu et al. have studied Nylon 6/Polypropylene (N6/PP) composites using Maleic anhydride (MA) as a grafting agent. They found that the notched impact strength, the stiffness and the strength of the alloys were higher than those in N6. They also found that the PP-g-MA showed a more homogeneous morphology and dispersion than the MA-free counterparts [22]. Additionally, Tand et al. have studied the N6/Polyphenylene Sulfide (N6/PPS) blend using SEBS-g-MA as the coupling agent. Their results indicated that the impact strength increased with the weight fraction of SEBS-g-MA increase, especially when the fraction was between $20 \%$ and 50\% [23]. Yang et al. have studied the fracture behavior of $\mathrm{N} 66 / \mathrm{TiO}_{2}$ composites. They showed that the toughness increased by $69 \%$ and $183 \%$, when the volume fraction of $\mathrm{TiO}_{2}$ was $1 \%$ and $3 \%$, respectively [24]. Later research showed the fracture toughness of the $\mathrm{N} 66 / \mathrm{TiO}_{2}$ composites was highly sensitive to temperature [25].

\subsubsection{Studies on crystallization behavior of the nylon composites}

Nylon (i.e., N66) contains polar amide bonds. Oxygen atom in the amide bond has high electronegativity and tends to form $\mathrm{H}$-bonds with hydrogen-containing polar materials. H-bond can also form between N66 molecular chains, which make crystallization of nylon easy [26, 27]. The structure of N66 is shown in Fig. 2.1. Filler introduction affects the crystallization behavior of nylon and therefore affects the composite mechanical properties. Grain refinement is known to be helpful in improving the toughness. 


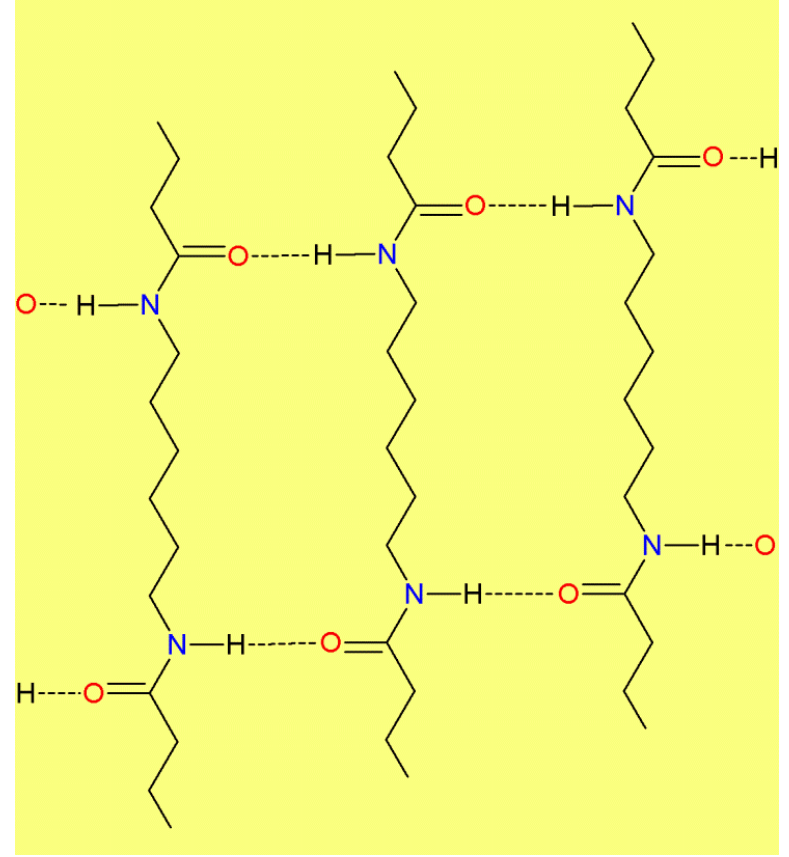

Figure 2.1: Schematic illustrating the N66 structure.

Bo et al. have studied the influence of different fraction of Montmorillonite (MMT) on N66 crystallization behavior. Their results showed that the MMT fraction between $2.04 \mathrm{wt} \%$ and $4.76 \mathrm{wt} \%$ gave the best crystallization behavior. They also indicated that crystallization rate of the composite was higher than that of N66 regardless of the MMT fraction [28]. The crystallization temperature reflected the overall crystallization rate due to the combined effects of nucleation and growth. Higher crystallization temperature $\left(\mathrm{T}_{\mathrm{c}}\right)$ would lead to lower super-cooling temperature $(\Delta \mathrm{T})$ and higher overall crystallization rate (Fig. 2.2).

Wang et al. have studied the crystallization behavior of N66/poly 2-hydroxy propylmethacrylate-methyl methacrylate/ $\mathrm{SiO}_{2}$ (N66/PHPMA-MMA/SiO ${ }_{2}$ ) composites using Differential Scanning Calorimetry (DSC). They made the conclusion that crystallization start 
temperature of hybrid material was higher than that of pure N66 and the crystallization temperature range was narrower [29]. Cooling method also made a significant impact on the crystallization behavior of the composites. Lu et al. have compared three different cooling ways of N66/clay composites. The three ways were melt-quench, post-annealing and melt-slow cooling annealing. Fourier Transform infrared spectroscopy (FTIR) spectroscopy and wide angle X-ray diffraction (XRD) showed that the melt-slow cooling-annealing was the most favorable way for crystallization. It yielded the highest crystallinity [30].

\subsubsection{Studies on performance of the nylon composites}

In recent years, studies on tribology, rheology, creep resistant and thermal degradation of nylon composites have gained increased attention. It is required that the composites can survive under harsh conditions such as oil-free lubrication, high-temperature ambient or overtime use.

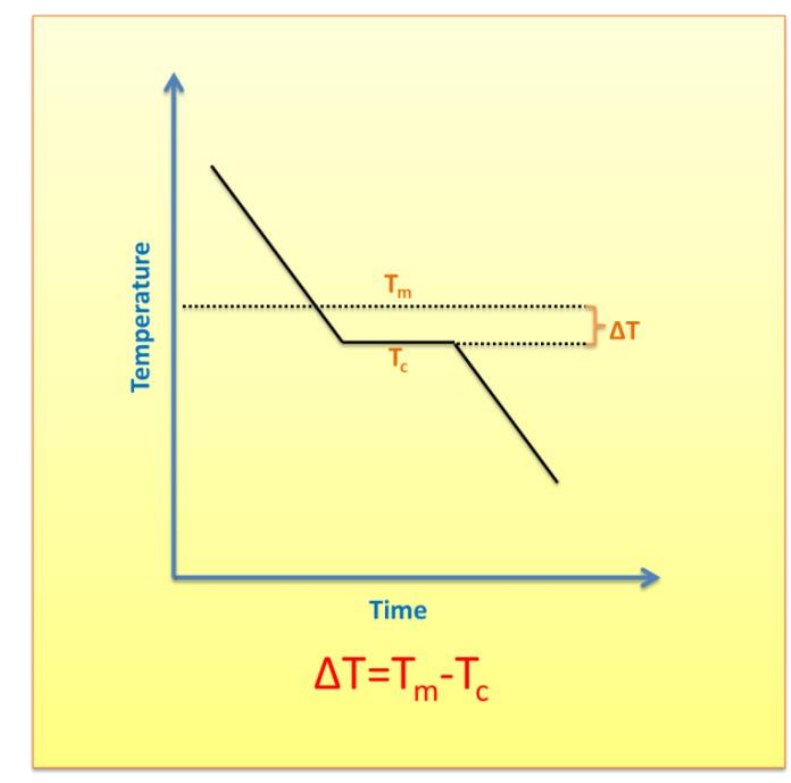

Figure 2.2: Schematic illustration of super-cooling. 
As described by Wang et al., addition of calcium sulphate whiskers (CWS) can decrease the friction coefficient of N66/Polyvinylidene Fluoride (N66/PVDF) composites and improve their wear resistance. They also suggested that the composite contained $5 \mathrm{wt} \% \mathrm{CSW}$ exhibited the best anti-wear ability [31]. In another study, Chen et al. have studied N66/PPS/Polytetrafluoroethylene (N66/PPS/PTEF) composites. They concluded that the friction coefficient of the composite minimized to 0.15 when the volume fraction of PTEF was $20 \%$ [32]. They also demonstrated that 80 vol\% of N66 with 20 vol\% of PPS yielded the lowest wear without PTEF [33]. Same group also studied the tribological behavior of N6/High Density Polyethylene (N6/DHPE) composites. They showed that the composites exhibited the best wear resistance when HDPE content reached $80 \%$ by volume [34].

Zhuang et al. have studied the tribology behavior of N66/MMT composites using cone and plate rheometers. They concluded that melt viscosity of the composite decreased with tremolite weight fraction increase from $10 \%$ to $50 \%$ [35]. Zhang et al. have studied the creep behavior of $\mathrm{N} 66 / \mathrm{TiO}_{2}\left(\mathrm{TiO}_{2}: 21 \mathrm{~nm}\right.$ particles $)$ using static tension and tensile creep tests. They found the nanoparticles accounted for a remarkable reduction in the creep rate under constant loading. They thought fillers may restrict the slippage, reorientation and motion of polymer chains and influence the stress transfer [36].

Ribeiro et al. have monitored the weight loss of N66/CNTs composites using Thermogravimetric Analysis (TGA). They found that the composite had the best thermal stability when the weight fraction of CNT was $0.5 \%$ or less [37]. Furthermore, Tatameshlou et al. have studied the decomposition of N66/Organic MMT (N66/OMMT) composites and found the 
decomposition temperature increased from $246{ }^{\circ} \mathrm{C}$ to $270{ }^{\circ} \mathrm{C}$ when weight fraction of OMMT was $4.1 \%[38]$.

\subsection{Localized surface plasmon resonance of silver nanowires}

Localized surface plasmon (LSP) modes are the collective oscillations of conduction electrons in nanostructures. These electron oscillations are due to restoring force of positive nuclei against displacement of the negatively-charged electrons. When the frequency of incident electromagnetic radiation matches the natural frequency of a LSP mode, the mode is excited in resonance (i.e., LSPR) [39, 40, 54]. The LSPR frequency shifts with nanostructure size/shape as well as the dielectric constant of the surrounding medium [40-42]. For a nanowire, symmetry is broken between longitudinal and transverse directions. Therefore, it has two SPR wavelengths: longitudinal and transverse. SPR occurs at a lower energy in longitudinal plasmon resonance because it is easier to polarize [43]. Both of the plasmon resonances are affected by the aspect ratio. However, the longitudinal plasmon resonance is more sensitive to aspect ratio change due to higher polarizability in the longitudinal direction [44]. When the aspect ratio of the AgNWs is sufficiently large, the plasmon resonances associated with the long and short axes are entirely decoupled. Therefore the peaks corresponding to LSPR of nanowires disappear [45]. It has been reported by Song et al. that no extinction peak associated with the longitudinal SPR is observable when the length of AgNWs is $2.5 \mu \mathrm{m}$ or longer [46]. Figure 2.3 illustrates the dipolar plasmon mode in the transverse direction. In addition, multipolar modes of plasmons can occur (i.e., quadrupole mode) with increasing nanowire cross-sectional area [55]. 


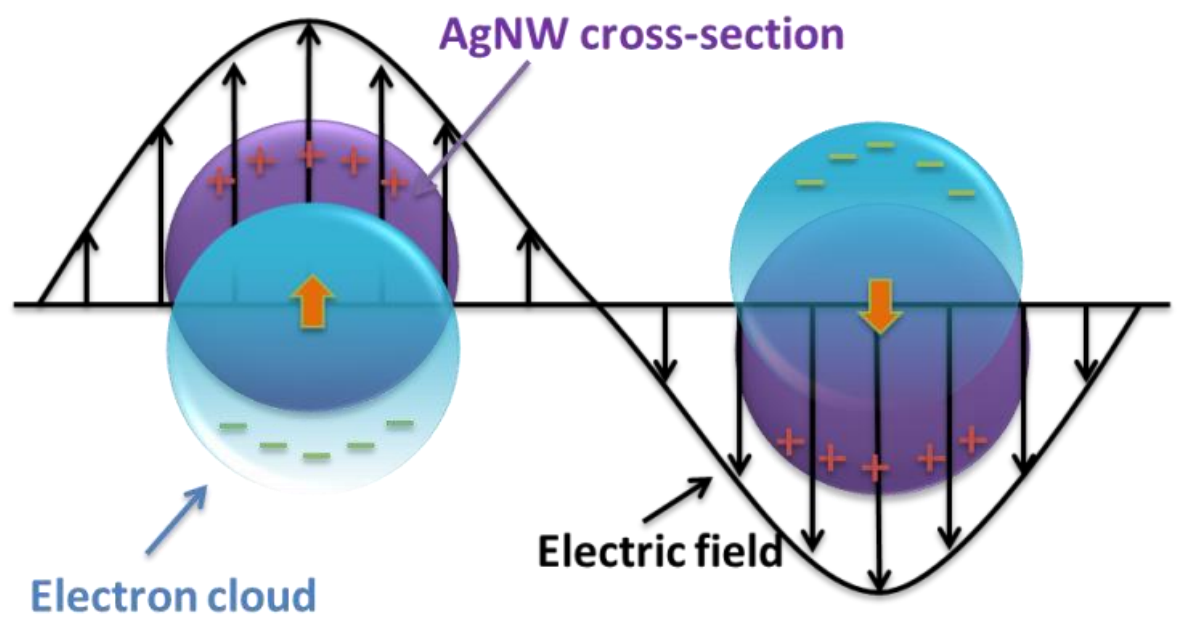

Figure 2.3: Schematic illustration of LSPR transverse electron oscillation for AgNWs.

\subsection{Polyol synthesis of AgNWs}

Polyol process involves reduction of an inorganic salt by a polyol at an elevated temperature. If polyol process also involves the use of a surfactant, which has selective coverage on different crystal planes, than directional growth can be achieved allowing the synthesis of NWs. For example, PVP is such a surfactant which selectively adsorbs to (100) atomic planes of Ag nanocrystals. Hence, it allows crystal growth only in the (111) atomic planes. As a result, directional growth of Ag nano-crystals occurs in the [111] direction leading to the formation of nanowires. Former researchers have proved that the PVP molecules are absorbed to the surface of the AgNWs through Ag:O coordination [47]. The key to the formation of wire-like nanometer sized structures is to form five-twinned nuclei though heterogeneous nucleation when $\mathrm{Ag}^{+}$is reduced to Ag seeds. Regular Ag nanoparticles will grow up from single-crystal nuclei instead of five-twinned nuclei. Some seeds under homogenous instead of heterogeneous nucleation will 
aggregate or be sintered to larger irregular nanoparticles [48]. Figure 2.4a illustrates the evolution process of various Ag nanocrystals ranging from $\mathrm{Ag}$ nanoparticles to nanowires. Figure $2.4 \mathrm{~b}$ illustrates the growing mechanism of AgNWs.
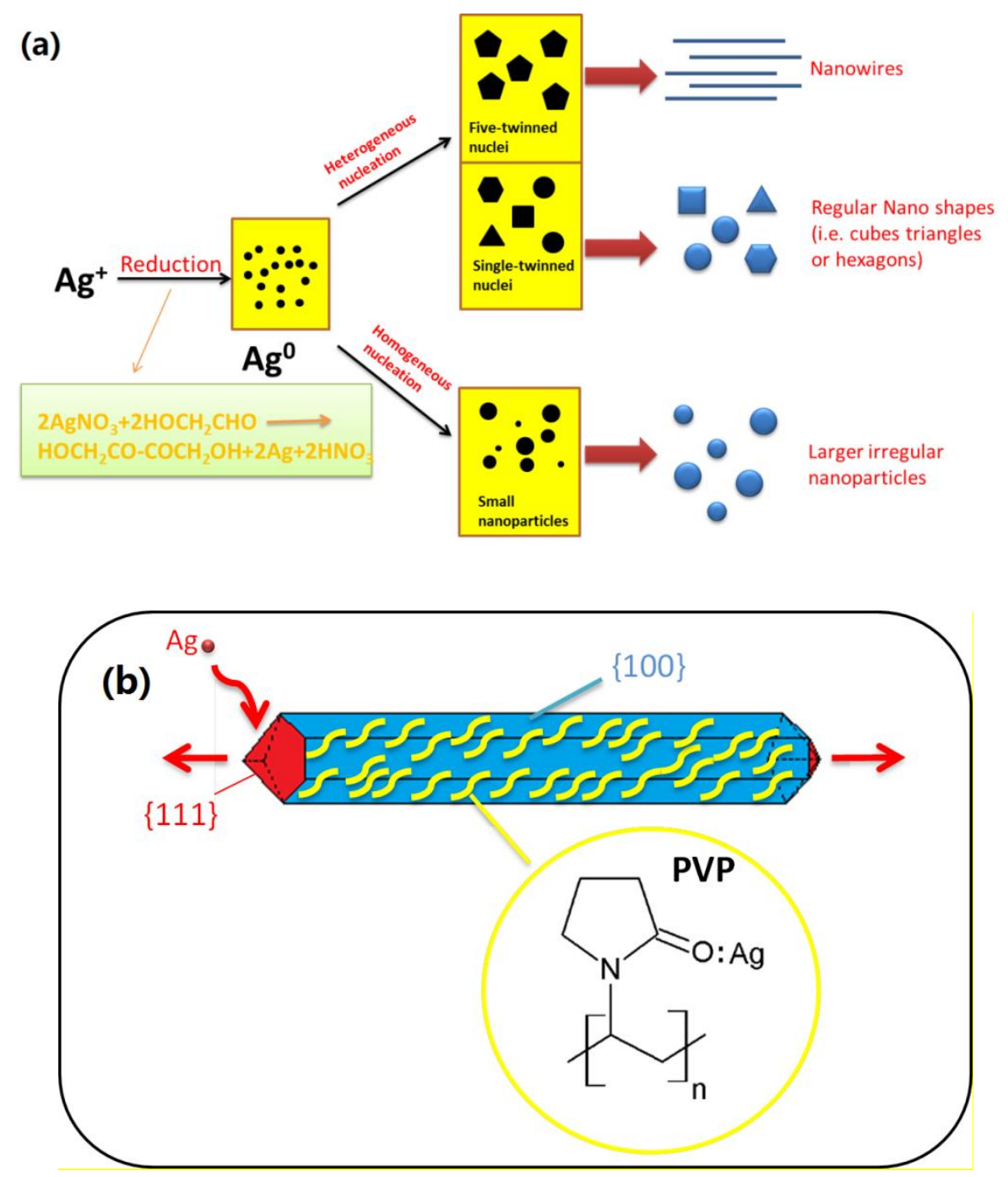

Figure 2.4: a) Schematics illustrating growth mechanisms of different Ag nanostructures. The sketch is inspired from Gao et al. [48]. b) Schematic illustration of the growing mechanism of AgNWs. The sketch is inspired from Mao et al. [47]. 
The nucleation modes can be controlled by polyol synthesis conditions. For example, surfactant or chloride ions may be introduced to prevent agglomeration of the seeds and prevent the seeds become larger due to Oswalt ripening. Morphologies and aspect ratios of the nanometer-sized structures could be varied from nanoparticles to long nanowires by adjusting the reaction time or temperature. Therefore, polyol process provides a simple and versatile approach to prepare 1D metal nanostructure. Typical factors affecting AgNW polyol synthesis are: $\mathrm{PVP} / \mathrm{AgNO}_{3}$ ratio, reaction temperature and seeding. Sun et al. have concluded that low $\mathrm{PVP} / \mathrm{AgNO}_{3}$ ratio may lead to thicker but longer nanowires due to incomplete coverage of PVP on the side surfaces. They suggested that the optimum $\mathrm{PVP} / \mathrm{AgNO}_{3}$ molar ratio was 1.5:1 [49]. They also found that the diameter of the AgNWs was reduced as the seed concentration in polyol solution was increased [50]. In addition, they indicated that the optimal temperature to yield maximum $\mathrm{AgNW}$ was $160{ }^{\circ} \mathrm{C}$ [47]. Later, same group found that $\mathrm{AgNO}_{3}$ reduction time also affected the morphology of AgNWs. TEM results showed that reduction time of $\mathrm{AgNO}_{3}$ should at least be $1 \mathrm{~h}$ to achieve wire-like shape [51]. However, those results partly contradicted with Gao et al.. They indicated that the reduction time of $\mathrm{AgNO}_{3}$ should be $41 \mathrm{~min}$ at $170{ }^{\circ} \mathrm{C}$. They claimed that morphologies of $\mathrm{AgNWs}$ remained almost unchanged but only elongated after a certain time [48]. The seeding condition of Sun's work was supported by Chen et al., as they demonstrated a relatively low concentration of nucleation is likely to result in more uniform nanowires than the high concentration case [52]. Later, Coskun et al. revealed that the factors which affected the morphologies of AgNWs were not comprehensive. They investigated the effects of $\mathrm{Cl}^{-}$ concentration, stirring rate and $\mathrm{AgNO}_{3}$ injection rate. Based on their results, the optimum 
parameters for $\mathrm{AgNW}$ synthesis are: $170{ }^{\circ} \mathrm{C}$ reaction temperature, 7.5:1 $\mathrm{PVP} / \mathrm{AgNO}_{3}$ molar ratio, $12 \mu \mathrm{M} \mathrm{NaCl}$ addition, $5 \mathrm{ml} / \mathrm{h} \mathrm{AgNO}_{3}$ injection rate and $1000 \mathrm{rpm}$ stirring rate [53]. 


\section{CHAPTER 3}

\section{Methodology}

This chapter presents the details of the methodology employed in both the synthesis of AgNWs and preparation/characterization of AgNW/N66 composites.

\subsection{AgNW preparation}

In the present study, AgNWs were synthesized by polyol synthesis. In this procedure, $\mathrm{AgNO}_{3}$ is used as the source of silver ions, $\mathrm{Ag}^{+}$, which are reduced by ethylene glycol (EG) at $170{ }^{\circ} \mathrm{C}$. EG also serves as the solvent as described in the paper of Coskun et al. [53]. The nanowires are washed and purified by centrifugation after the synthesis.

\subsubsection{Synthesis of AgNWs}

In this process, $4.995 \mathrm{~g}$ of PVP (MW=55,000, Sigma-Aldrich) was dissolved in $100 \mathrm{ml}$ of ethylene glycol (EG) by vortex mixing for 2 min and stored in a plastic bottle with air tight seal for at least one day before use to make sure the PVP is completely dissolved. Next, $5 \mathrm{~g}$ of $\mathrm{AgNO}_{3}$ is dissolved in $245 \mathrm{ml}$ of $\mathrm{EG}$ at a concentration of $0.12 \mathrm{M}$. The $\mathrm{AgNO}_{3}$ solution was stored in an amber glass bottle in the refrigerator. After preparation, the solution was kept for at least one day before use. Subsequently, $20 \mathrm{ml}$ of the PVP solution (in EG) was transferred to a $100 \mathrm{ml}$ flask. The flask was pre-cleaned by DI water (50\%) + isopropyl alcohol (IPA) (50\%) mixture and ultrasonicated for $1 \mathrm{~h}$. The flask was then blow-dried by argon. Next, $28 \mathrm{mg}$ of $\mathrm{NaCl}$ was added into 
the flask containing PVP dissolved in EG. The solution was ultra-sonicated for 2 min to dissolve the solid $\mathrm{NaCl}$. Then, the flask was placed in a heating mantle having both heating and magnetic stirring capabilities. A condenser was assembled on the neck of the flask with cold water running from the bottom to the top. The condenser was supported by an iron stand. The setup for the synthesis of AgNWs is shown in Fig. 3.1.

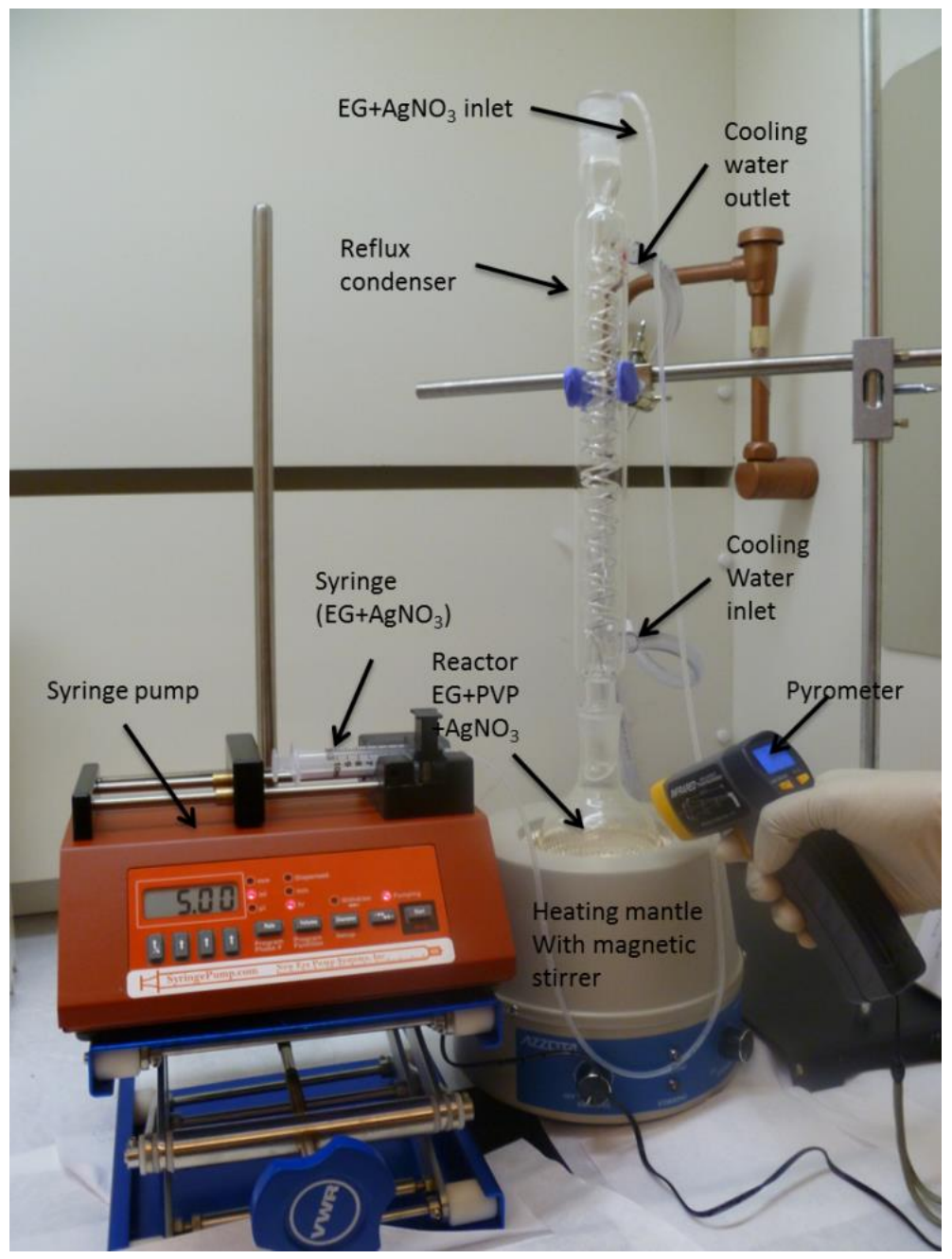

Figure 3.1: Photograph of the experimental setup for the synthesis of AgNWs (taken by Sriharsha Karumuri). 
Prior to the initiation of $\mathrm{Ag}$ reduction, the PVP solution was kept at $170{ }^{\circ} \mathrm{C}$ for $1 \mathrm{~h}$ under continuous stirring using a star-head magnetic stir bar $(0.68 " \times 0.56$ ") at $1000 \mathrm{rpm}$. The solution temperature was monitored by a liquid thermometer. Subsequently, AgNW reduction was initiated by dropwise addition of $0.12 \mathrm{M} \mathrm{AgNO}_{3}$ into the PVP solution by Era NE-300 syringe pump at a rate of $20 \mathrm{ml} / \mathrm{h}$. The injection was continued for $1 \mathrm{~h}$. After $\mathrm{AgNO}_{3}$ injection is complete, the resulting nanowire suspension was further stirred at $170{ }^{\circ} \mathrm{C}$ for $30 \mathrm{~min}$. Finally, the suspension was air-cooled to room temperature.

\subsubsection{Separation and washing of AgNWs}

AgNW suspension prepared by polyol process contains excess PVP. In order to remove the excess PVP and transfer the AgNWs into water, the suspension was dispersed in acetone + ethanol and centrifuged. Acetone and ethanol was purchased from Oklahoma State University Chemical Store and used without further purification.

Five $\mathrm{ml}$ of $\mathrm{AgNW}$ suspension was put in a centrifuge tube and diluted 5 times by acetone and centrifuged twice at 3,500 rpm for $30 \mathrm{~min}$. After the first centrifugation, three different layers formed. The supernatant was acetone. The middle layer being yellow was the excess PVP, which was removed by a pipette. Silver nanowires were separated from the suspension in the form of a

precipitate. Subsequently, the supernatant was removed and the precipitate was dispersed in 30 $\mathrm{ml}$ acetone and again centrifuged at $3500 \mathrm{rpm}$ for $30 \mathrm{~min}$. Next, the nanowires were dispersed in ethanol and centrifuged at $3500 \mathrm{rpm}$ for $30 \mathrm{~min}$. After three centrifugations, AgNWs were characterized by UV-Vis spectroscopy and the extinction spectrum was checked to assure reproducibility. 


\subsection{AgNW/N66 composite preparation}

\subsubsection{Conventional interfacial polycondensation of N66}

In this section, interfacial polycondensation of N66 is described. The polycondensation reaction employs two monomers: 1,6-diaminohexane in water and adipoyl chloride dissolved in nonpolar organic solvent. A protonated amide is formed as soon as the two solutions are mixed. The proton is rapidly eliminated and combines with $\mathrm{Cl}^{-}$forming $\mathrm{HCl}$. The polymer forms near the interface but at the organic solvent side, because adipoly chloride is essentially not soluble in water while the diaminohexane is partially soluble in the organic solvent. Therefore, acylation reaction occurs in the organic solvent close to the interface. Figure 3.2 illustrates the relationship of reactants in interfacial polycondensation. Accumulation of the polymer product at the interface prevents diaminohexane transport and limits the reaction. Hence, in order to maintain polycondensation, the polymer accumulating at the interface must be continuously removed.

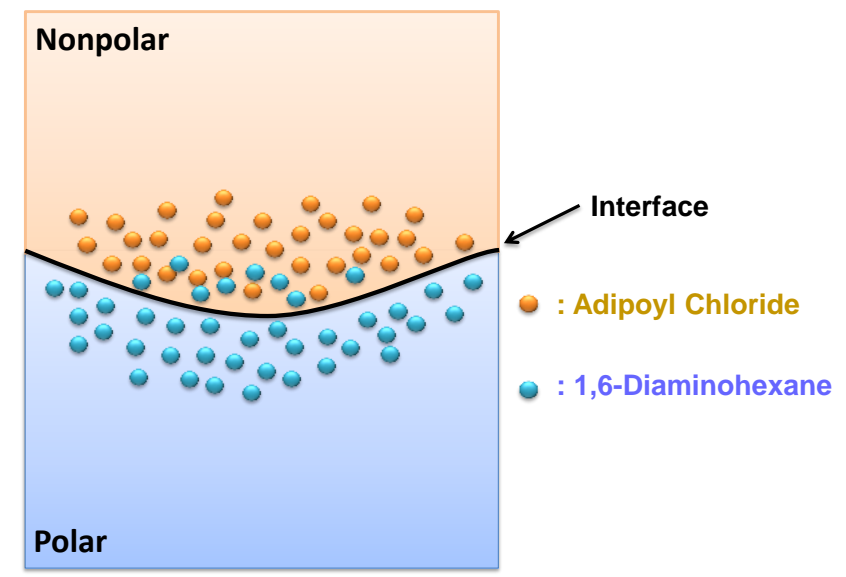

Figure 3.2: Schematic illustration of interfacial polycondensation. The sketch is inspired from Morgan et al. [16]. 


\subsubsection{AgNW/N66 composite synthesis}

Hundred $\mathrm{g}$ of 1,6-diaminohexane was dissolved in $1250 \mathrm{ml}$ of water by stirring for 30 min using a magnetic stir bar $\left(8 \times 15.9 \mathrm{~mm}^{2}\right)$. Next, $0.065 \mathrm{~g}$ of AgNWs (based on the calculation) was dispersed in $120 \mathrm{ml}$ of THF by vortex mixing for $5 \mathrm{~min}$ to form AgNW suspension. The AgNW suspension was stored in an amber bottle. Subsequently, $20 \mathrm{ml}$ of this AgNW suspension was transferred to a $50 \mathrm{ml}$ centrifuge tube and $1 \mathrm{ml}$ of adipoyl chloride (as received) was injected to the suspension using a pipette. Next, $20 \mathrm{ml}$ of 1.6-diaminohexane (prepared as above) solution was added to the suspension. The mixture was vortex mixed for $10 \mathrm{~s}$. Polycondensation started once 1.6-diaminohexane was added to the suspension where AgNW/N66 composite microparticles formed. The mixture was left in the hood for $12 \mathrm{~h}$ to complete the polycondensation reaction. Experiment procedures of AgNW/N66 composite synthesis and separation are shown in Fig. 3.3.

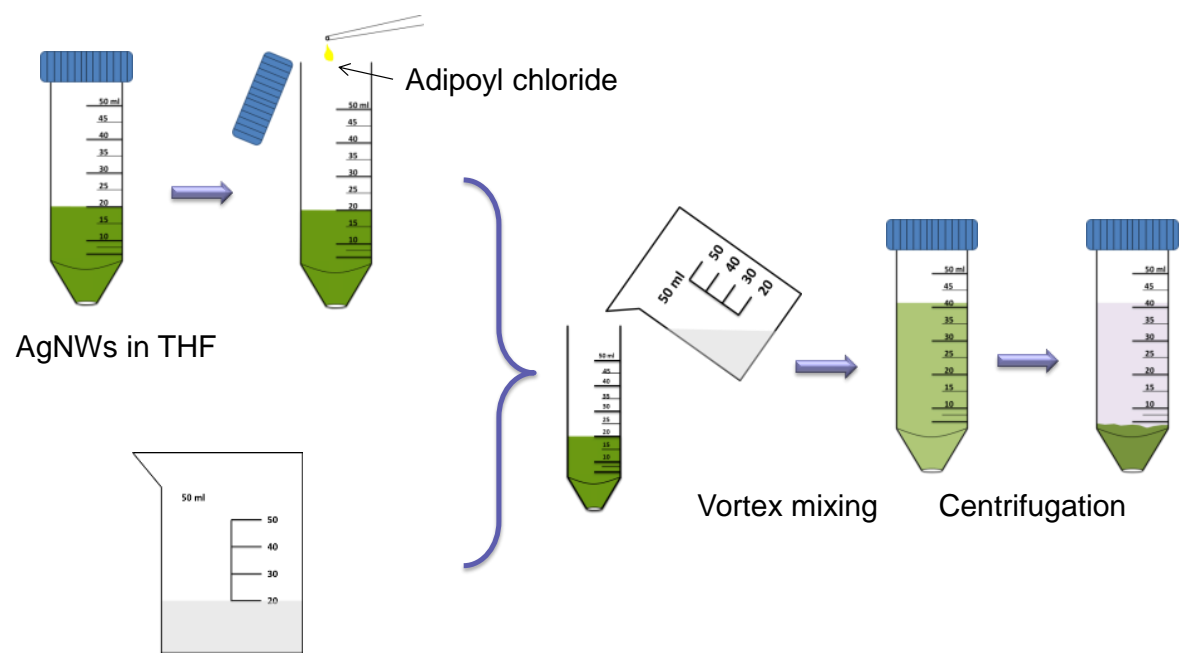

1,6-diaminohexane in water

Figure 3.3: Schematic illustration of AgNW/N66 composite synthesis and separation. 
1,6-diaminohexane (98+\%) and adipoyl chloride (98\%) were purchased from Alfa Aesar, USA. THF was purchased from Oklahoma State University Chemical Store. All the chemicals were used without further purification.

\subsubsection{Separation and cleaning of AgNW/N66 microparticles}

After the completion of polycondensation, the AgNW/N66 composite microparticles were separated from the liquid and dried. The separation was achieved by centrifugation at 2,600 rpm for 10 min. After the centrifugation, supernatant was removed and the particles were dispersed in $30 \mathrm{ml}$ of DI water by vortex mixing for $20 \mathrm{~s}$. Subsequently, another centrifuge step was run as previously and the particles were transferred to a glass vial. Finally, the residual water was evaporated by placing the vial on a hot plate at $45^{\circ} \mathrm{C}$ for 5 days.

\subsection{Theoretical calculation of silver weight fraction in $\mathbf{N 6 6}$}

In order to calculate the weight fraction of $\mathrm{Ag}$ in the composites, we assume all the $\mathrm{Ag}^{+}$ in $\mathrm{AgNO}_{3}$ is reduced to $\mathrm{Ag}$ by $\mathrm{EG}$ at $170{ }^{\circ} \mathrm{C}$. Given the concentration and volume of the $\mathrm{AgNO}_{3}$ solution, the weight of $\mathrm{Ag}$ is obtained. Subsequently, the weight of the composite is acquired by an electronic scale. Thus, Ag weight fraction is calculated by Ag mass divided by composite mass. Different Ag fraction composites are obtained by controlling the Ag weight in the composites. Complete incorporation of nanostructured Ag into N66 is checked by UV-Vis spectroscopy assuring no measurable Ag remains in the solvent after polymerization.

\subsection{Casting of AgNW/N66 samples}


After dried, the composite microparticles were transferred into a glass vial. The glass vial was sealed by an aluminum cap with a Teflon septum. Subsequently, the vial was purged with argon and lowered into a melter by glass wire. The sample was melt at $265{ }^{\circ} \mathrm{C}$ for $20 \mathrm{~min}$ and then cooled to room temperature. A K-type thermocouple was used to monitor the temperature. The setup for the sample casting is shown in Fig. 3.4a. Figure 3.4b shows the glass vial sealed with an aluminum cap and Teflon septa. After solidification and cooling, the glass vial was broken to release the solid composite sample. For the nano-indentation measurements, the samples were grinded to squares $\left(4 \times 4 \times 1.3 \mathrm{~mm}^{3}\right)$ as shown in Fig. 3.5.

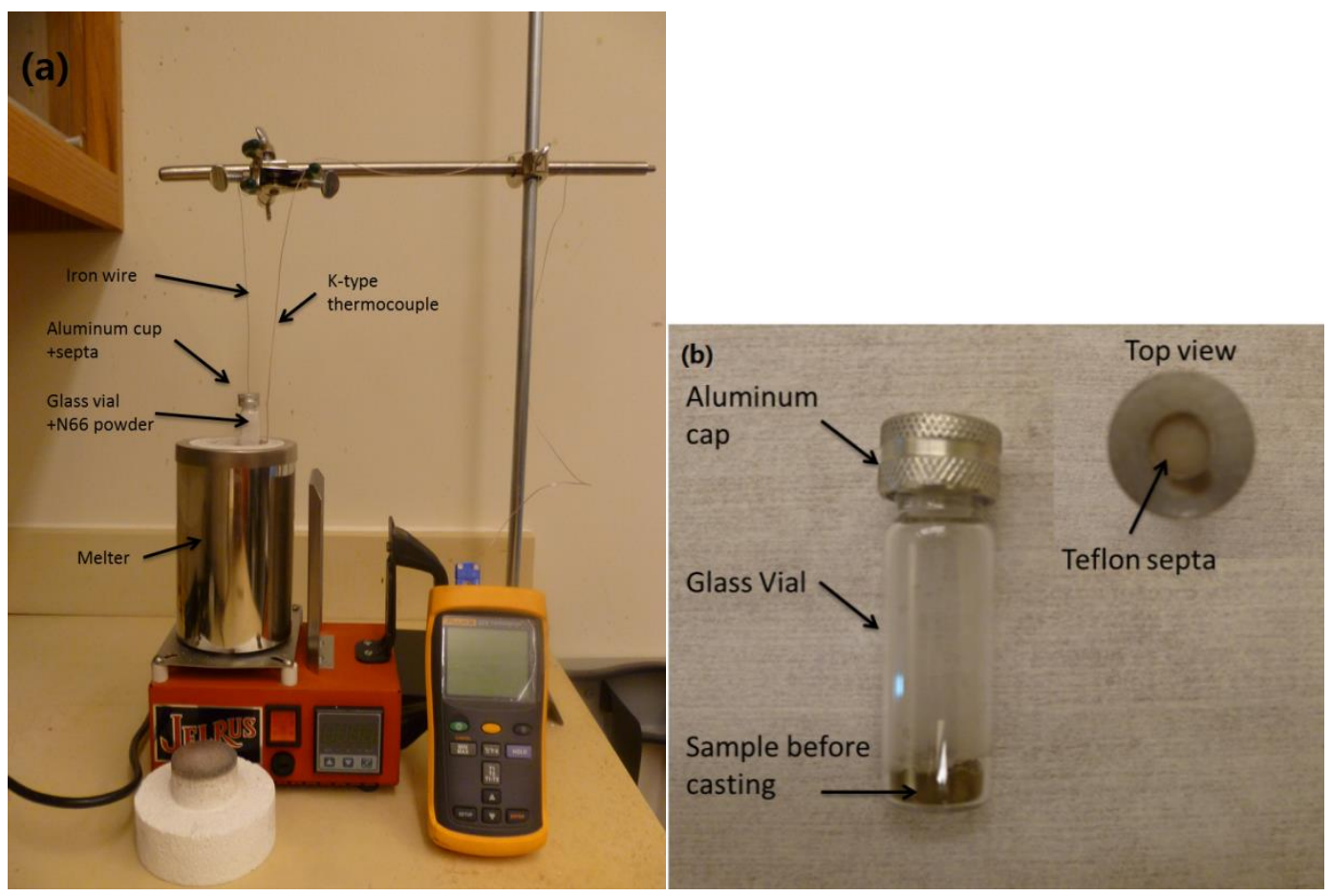

Figure 3.4: a) Photograph illustrating the sample casting. b) Glass vial with aluminum cap and Teflon septum. 


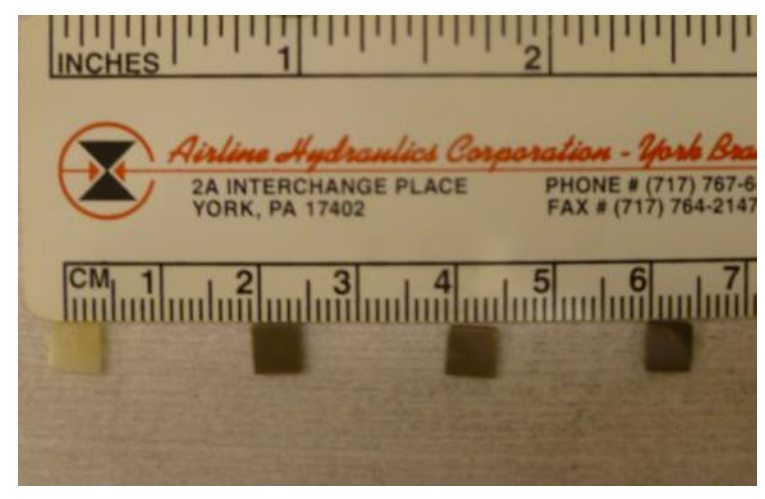

Figure 3.5: AgNW/N66 samples prepared for nano-indentation. Ag weight fraction from left to right is $0 \%, 1.49 \%, 3.28 \%$ and $6.74 \%$, respectively.

\subsection{Characterization of the composites}

\subsubsection{UV-Vis spectroscopy}

After the synthesis of AgNWs (Section 3.1.1), their extinction spectrum was acquired by UV-Vis spectroscopy. The scans were recorded using a Cary 300 UV-Vis spectrophotometer. Quartz optical cells with an optical length of $1 \mathrm{~cm}$ were employed. AgNWs were diluted 120 times in EG before transferred into the optical cells. Pure EG was used as the reference. A typical scan was started at wavelength of $800 \mathrm{~nm}$ and ended at $200 \mathrm{~nm}$ with data intervals of $1 \mathrm{~nm}$. Scan rate was set at $600 \mathrm{~nm} / \mathrm{min}$. The extinction spectrum of the AgNWs was used to assure reproducibility.

\subsubsection{Scanning electron microscopy}

The morphology of the AgNWs was studied by FEI Quanta 600 field-emission scanning electron microscope (SEM). SEM was operated at an accelerating voltage of $20 \mathrm{kV}$. 


\subsubsection{X-ray diffraction}

$\mathrm{X}$-ray diffraction (XRD) was performed to determine the variation of mass ratio between $\mathrm{Ag}$ and N66. XRD patterns were recorded using a Philips Analytical X-Ray PW 3710 diffractometer. The diffractometer was employed with $\mathrm{CuK}_{\alpha}$ radiation source, a tube voltage of $40 \mathrm{kV}$ and tube current of $40 \mathrm{~mA}$. Spectra were scanned in the range of $2 \theta$ from $10^{\circ}$ to $90^{\circ}$ at a rate of $2 \%$ min.

\subsubsection{Raman spectroscopy}

Raman scattering provides useful chemical information. Raman signal of the AgNWs and PVP were acquired with a Witec-alpha 300R Raman microscope with $532 \mathrm{~nm}$ laser as the excitation source. The dried AgNW/N66 composite microparticles and PVP powder were placed on a microscope slide and measured in dark environment at room temperature. The Raman spectra were collected under $0.5 \mathrm{~mW}$ incident laser power with $20 \mu \mathrm{m}$ spot size. Graphical illustration of a Raman acquisition is shown in Fig. 3.6.

\subsubsection{Fourier transform infrared spectroscopy}

In addition to Raman spectroscopy, Fourier Transform Infrared Spectroscopy (FTIR) was also conducted for chemical analysis. FTIR was performed using Nicolet iS50 in ATR mode with a diamond probe. The sample powders were loaded on the FTIR cell above the infrared detector by pressing using an applicator. We employed $2 \mathrm{~cm}^{-1}$ spectral resolution and 64 scans to average.

The FTIR spectra were recorded in the range of $500-3500 \mathrm{~cm}^{-1}$. 


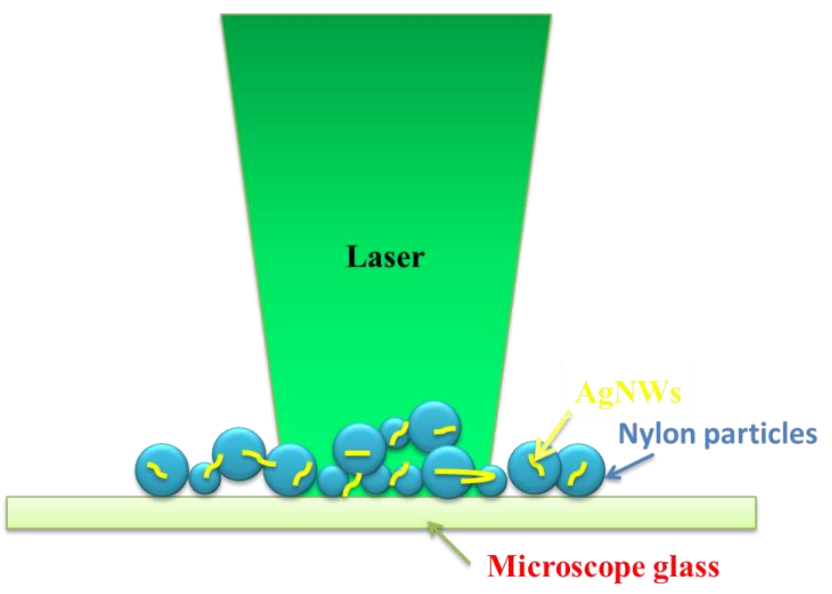

Figure 3.6: Graphical illustration of a Raman acquisition.

\subsubsection{Differential scanning calorimeter}

Melting and crystallization profiles of the solid samples were derived using a TA Q2000 differential scanning calorimeter (DSC) in nitrogen ambient. Powder samples of $10 \mathrm{mg}$ were filled in standard DSC aluminum pans. Prior to the measurement, the temperature was elevated to $285{ }^{\circ} \mathrm{C}$ at a ramping rate of $10{ }^{\circ} \mathrm{C} / \mathrm{min}$ to release the heat residue of the samples. Then, measurement was started by cooling at $10{ }^{\circ} \mathrm{C} / \mathrm{min}$ to $-30{ }^{\circ} \mathrm{C}$. Subsequently, the measurement was continued in the heating mode at a temperature ramp of $10{ }^{\circ} \mathrm{C} / \mathrm{min}$ to $250^{\circ} \mathrm{C}$.

\subsubsection{Nano-indentation}

Mechanical properties of AgNW/N66 composites were studied using nano-indentation. An MTS Nano Indenter XP Elastic was employed in obtaining the modulus and hardness of 
composites with a maximum load of $0.98 \mathrm{mN}$ using a Berkovich indenter tip with a half angle of $70.3^{\circ}$. Test performed on each sample constituted 36 indentations in a $6 \times 6$ matrix format. 


\section{CHAPTER 4}

\section{Results and Discussion}

\subsection{Structural characterization of AgNWs}

Silver nanowires were synthesized as described in Sections 3.1.1 and 3.1.2. During the synthesis process, solution color changed from light yellow to orange and then to hazy greyish brown. The final product was a mixture of AgNWs and silver nanoparticles (AgNPs). Subsequently, the AgNWs were separated from the AgNPs using centrifugation. Therefore, the characterized suspensions had low fraction of AgNPs. The UV-Vis spectroscopy was conducted as described in Section 3.5.1. Figure 4.1 shows the average optical extinction spectrum of the AgNW suspensions. The spectrum exhibits two relatively sharp LSPR peaks at about 350 and $390 \mathrm{~nm}$ with a broad tail towards longer wavelength. The broad tail is due to the distribution of different sizes and shapes [54]. The peaks at 350 and $390 \mathrm{~nm}$ are attributed to the out-of-phase quadrupolar and transverse dipolar LSPR in AgNWs, respectively [47].

Morphology of the prepared AgNWs was studied by SEM as described in Section 3.5.2. The SEM image in Fig. 4.2 shows that the AgNWs are 5-10 $\mu \mathrm{m}$ long and have diameter of $43 \pm 15$ nm. The image also shows that AgNPs as well as the silver nanorods (AgNRs) cannot be completely removed by centrifugation. 


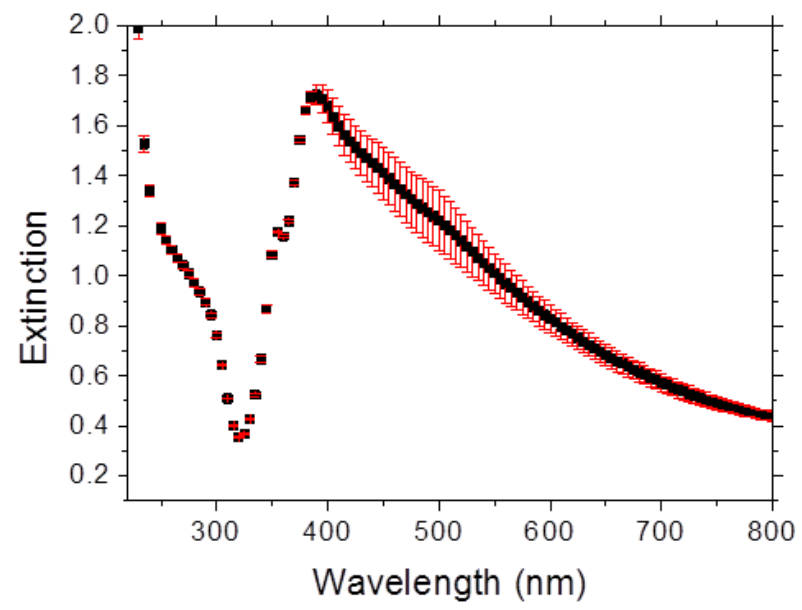

Figure 4.1: Average optical extinction spectrum of the AgNWs after 8 batches with error bars.

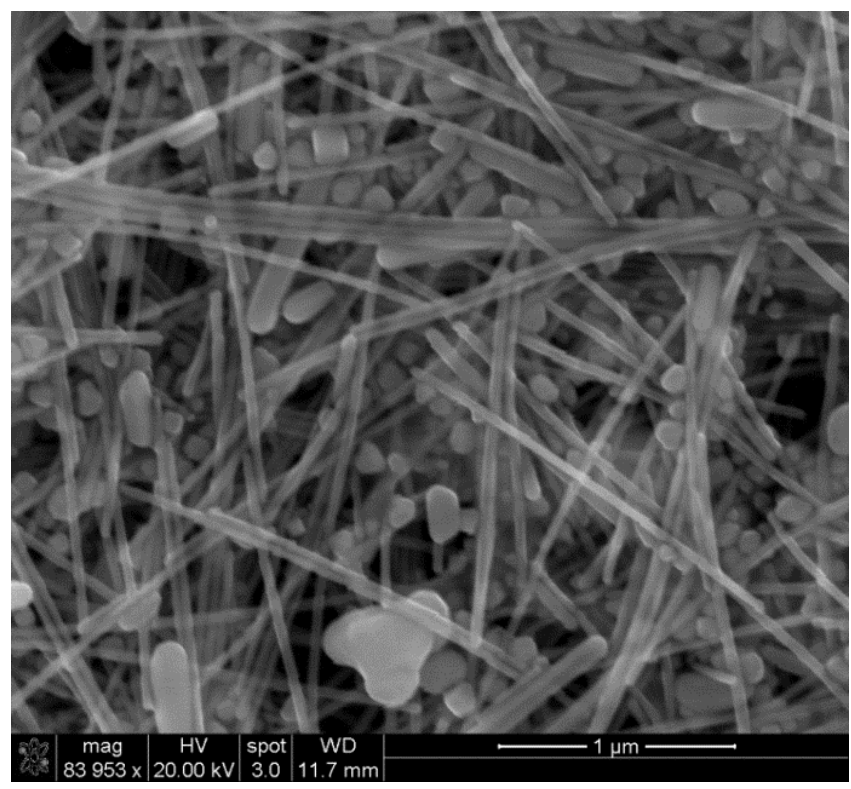

Figure 4.2: SEM image of the AgNWs synthesized by the polyol process.

The AgNWs, which are functionalized by PVP were characterized by Raman spectroscopy as described in Section 3.5.4. Fig. 4.3a shows the Raman spectrum of the neat PVP 
for comparison. Assignments of the Raman peaks in the PVP spectrum are as follows. The peak at $756 \mathrm{~cm}^{-1}$ corresponds to $\mathrm{C}-\mathrm{N}$ bending [56]. The $934 \mathrm{~cm}^{-1}$ peak is assigned to $\mathrm{C}-\mathrm{C}$ stretching in the pyrrolidone ring [57]. The band at $1233 \mathrm{~cm}^{-1}$ is due to $\mathrm{C}-\mathrm{N}$ stretching. The $1429 \mathrm{~cm}^{-1}$ peak is assigned to $\mathrm{C}-\mathrm{H}$ bending in the backbone chain $[56,57]$. The band at $1670 \mathrm{~cm}^{-1}$ corresponds to $\mathrm{C}=\mathrm{O}$ stretching. The $2927 \mathrm{~cm}^{-1}$ peak is attributed to $\mathrm{C}-\mathrm{H}$ stretching in $\mathrm{CH}_{2}$ in the pyrrolidone ring [56]. A weak shoulder at $2983 \mathrm{~cm}^{-1}$ is due to asymmetric $\mathrm{C}-\mathrm{H}$ stretching in $\mathrm{CH}_{2}$ in the backbone chain [57].

Figure $4.3 \mathrm{~b}$ shows the Raman spectrum of the AgNWs (functionalized by PVP). Compared with the PVP, the AgNWs (functionalized by PVP) exhibit a distinguishing peak at $239 \mathrm{~cm}^{-1}$ indicative of $\mathrm{Ag}-\mathrm{O}$ stretching [47]. This peak is significantly enhanced (due to surfaceenhanced Raman scattering (SERS)), implying coordination of the PVP with the Ag surface through the lone pair electrons of the oxygen atom in the carbonyl group. Meanwhile, the $\mathrm{C}=\mathrm{O}$ stretching vibration shifts from 1670 to $1590 \mathrm{~cm}^{-1}$, verifying PVP molecules bind with AgNW through oxygen in the $\mathrm{C}=\mathrm{O}$ group [47]. The enhanced peak at $2937 \mathrm{~cm}^{-1}$ is attributed to asymmetric $\mathrm{C}-\mathrm{H}$ stretching in $\mathrm{CH}_{2}$ in the backbone chain of PVP. Accordingly, it is inferred that the $\mathrm{CH}_{2}$ chains are close to the surface of AgNWs [47] accounting for SERS. An additional attribute of the Ag is seen to be significant lowering of the fluorescence background of PVP due to resonant energy transfer from PVP to Ag nanostructures. 

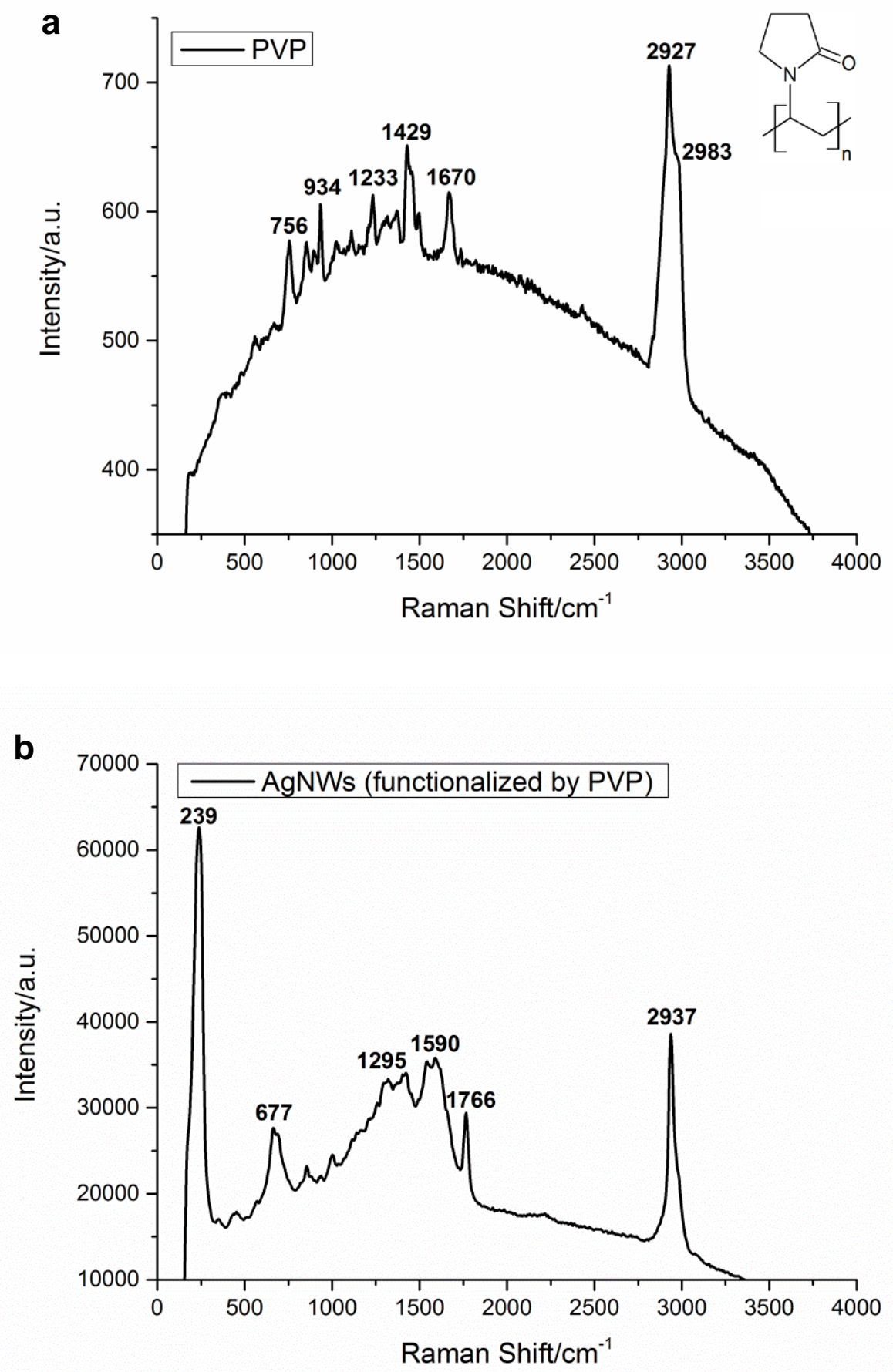

Figure 4.3: a) Raman spectrum of PVP. b) Raman spectrum of AgNWs (functionalized by PVP). 


\subsection{Physical characterization of AgNW/N66 composite microparticles}

The AgNW/N66 composite microparticles were prepared as described in Section 3.2.2. Representative particles are shown in Fig. 4.4. Darkening of the color is observed when weight fraction of Ag is increased in the composite samples. The morphology of the microparticles was first investigated using an optical microscope in bright field (Fig. 4.5). The particle shape is found to be approximately spherical with an average diameter of $7 \mu \mathrm{m}$.

The morphology of the AgNW/N66 composite microparticles was further studied by SEM (Fig. 4.6). Insignificant amount of AgNWs as well as the AgNPs can be seen on the surface of the N66 microparticles. In addition, when polycondensation reaction was completed, the supernatant was transparent, implying no AgNWs were left in the supernatant. Therefore, we infer that majority of AgNWs are embedded in N66 during the polycondensation reaction.

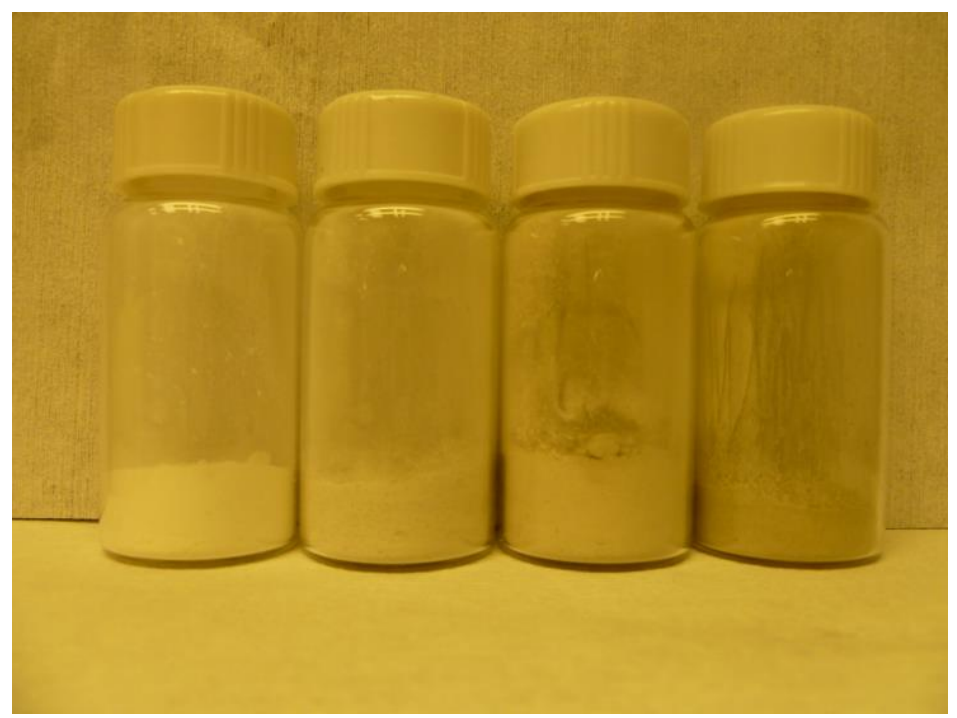

Figure 4.4: $\mathrm{AgNW} / \mathrm{N} 66$ composite microparticles. Weight fraction of $\mathrm{Ag}$ from left to right is $0 \%$, $1.49 \%, 3.28 \%$ and $6.74 \%$. 


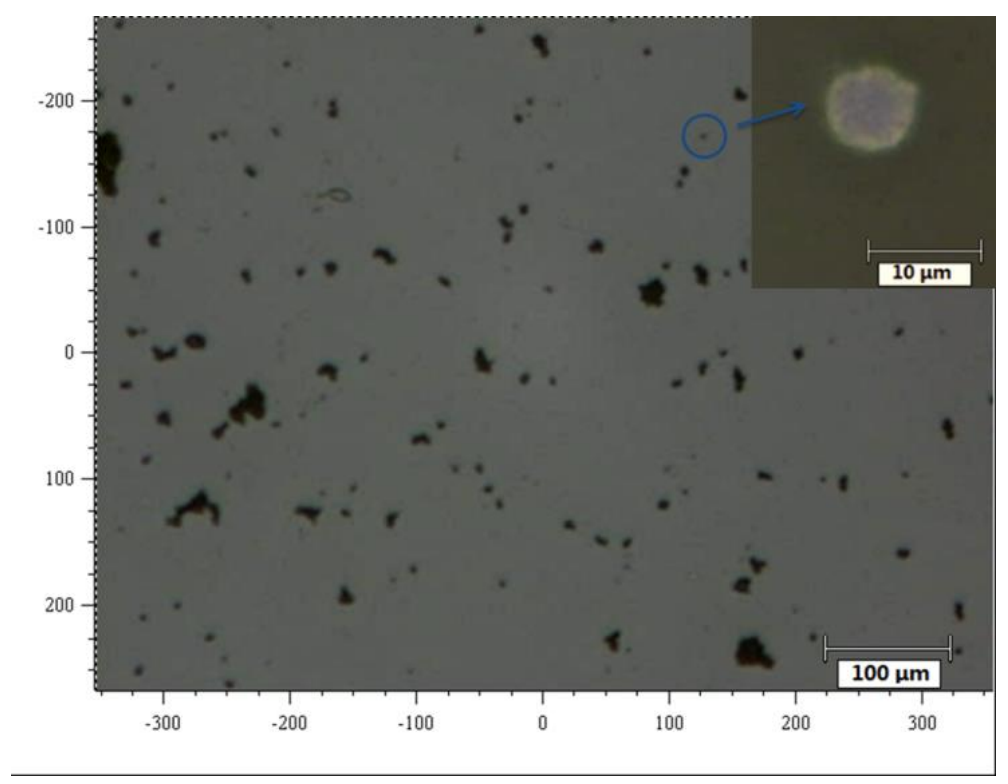

Figure 4.5: Optical microscope image of AgNW/N66 microparticles.

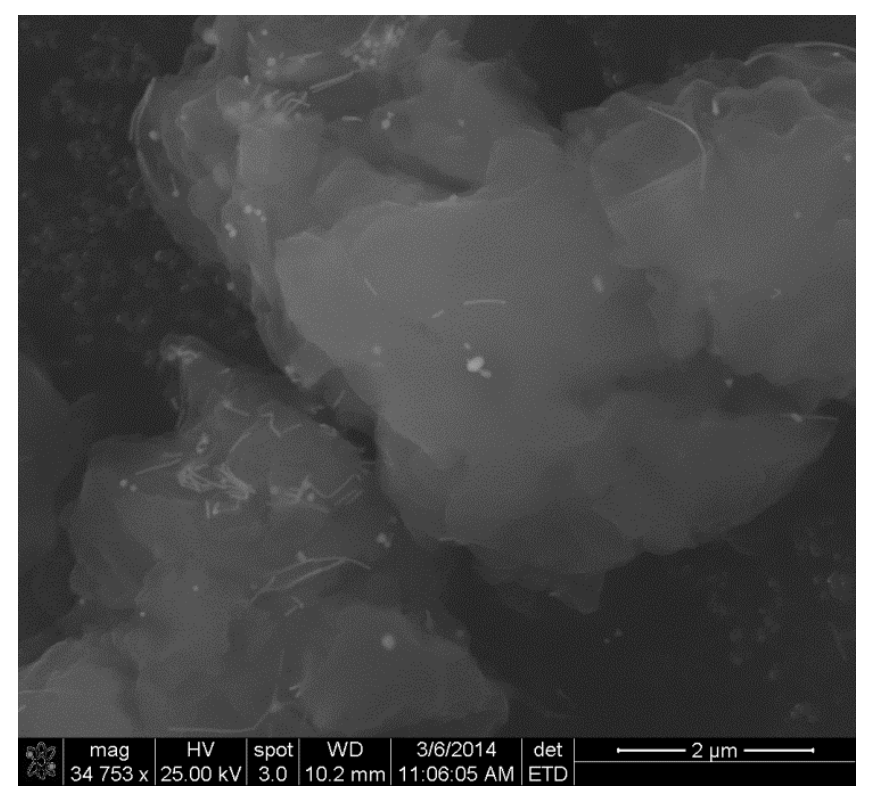

Figure 4.6: SEM image of AgNW/N66 composite microparticles. 


\subsection{Fourier Transform Infrared Spectroscopy}

AgNW/N66 composite microparticles were studied by FTIR spectroscopy as described in Section 3.5.5. FTIR spectra of commercial N66 and N66 synthesized in the present work are shown in Fig. 4.7.

Assignments of FTIR peaks in the spectra of N66 are as follows. The peaks at 3298 and $2932 \mathrm{~cm}^{-1}$ are due to $\mathrm{N}-\mathrm{H}$ and $\mathrm{C}-\mathrm{H}$ stretching [58], respectively. The peaks at 1630 and 1534 $\mathrm{cm}^{-1}$ corresponds to $\mathrm{C}=\mathrm{O}$ and $\mathrm{C}-\mathrm{N}$ stretching [58]. Finally, the peak at $1139 \mathrm{~cm}^{-1}$ is assigned to C-C stretching [59]. The FTIR spectra of the commercial N66 and N66 in this work are essentially the same.

FTIR spectra of the PVP and the AgNW/N66 composite particles are shown in Fig. 4.8. Assignments of the FTIR peaks in the spectra of PVP are as follows. The peak at $733 \mathrm{~cm}^{-1}$ corresponds to the $\mathrm{C}-\mathrm{C}$ stretching in the backbone chain [57]. The $843 \mathrm{~cm}^{-1}$ peak is assigned to $\mathrm{C}-\mathrm{C}$ stretching in the pyrrolidone ring [57]. The peak at $1248 \mathrm{~cm}^{-1}$ is due to $\mathrm{C}-\mathrm{N}$ stretching [60]. The peak at $1421 \mathrm{~cm}^{-1}$ corresponds to $\mathrm{C}-\mathrm{H}$ bending [57]. The peak at 1652 is assigned to $\mathrm{C}=\mathrm{O}$ stretching [60]. The peak at $2886 \mathrm{~cm}^{-1}$ is due to $\mathrm{C}-\mathrm{H}$ stretching in the backbone chain [57]. The peaks located at 2919 and $2948 \mathrm{~cm}^{-1}$ are assigned to $\mathrm{C}-\mathrm{H}$ stretching in $\mathrm{CH}_{2}$ in the backbone chain and pyrrolidone ring [57], respectively. The peak located at $3446 \mathrm{~cm}^{-1}$ is due to $\mathrm{O}-\mathrm{H}$ stretching vibration [60]. It indicates the presence of hydroxyl groups in physisorbed water. This finding agrees with Giri et al. [60]. 


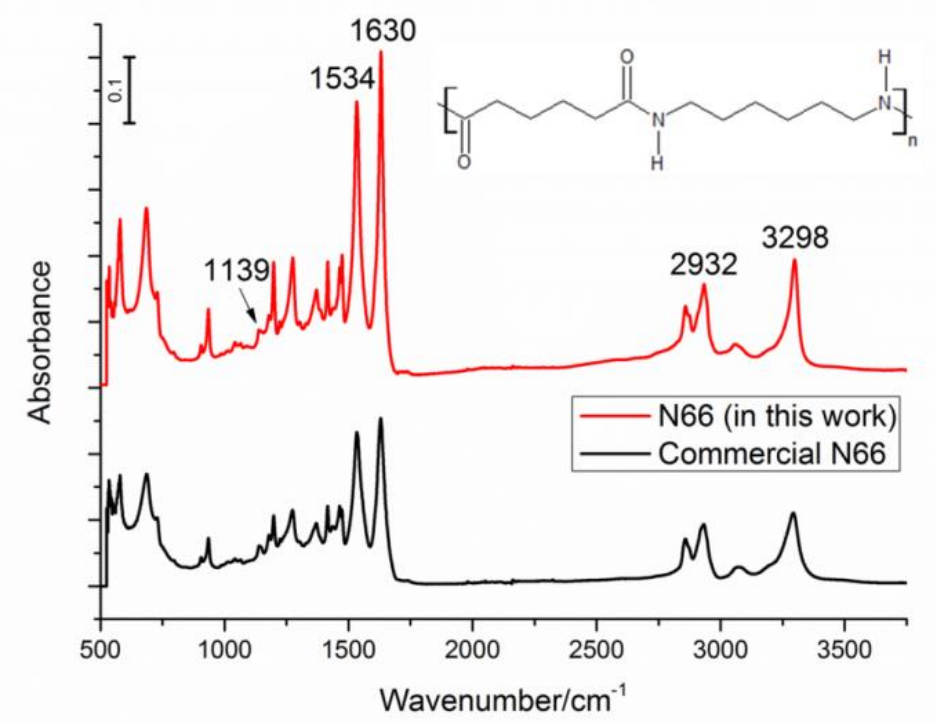

Figure 4.7: FTIR spectra of the commercial N66 and N66 in this work.

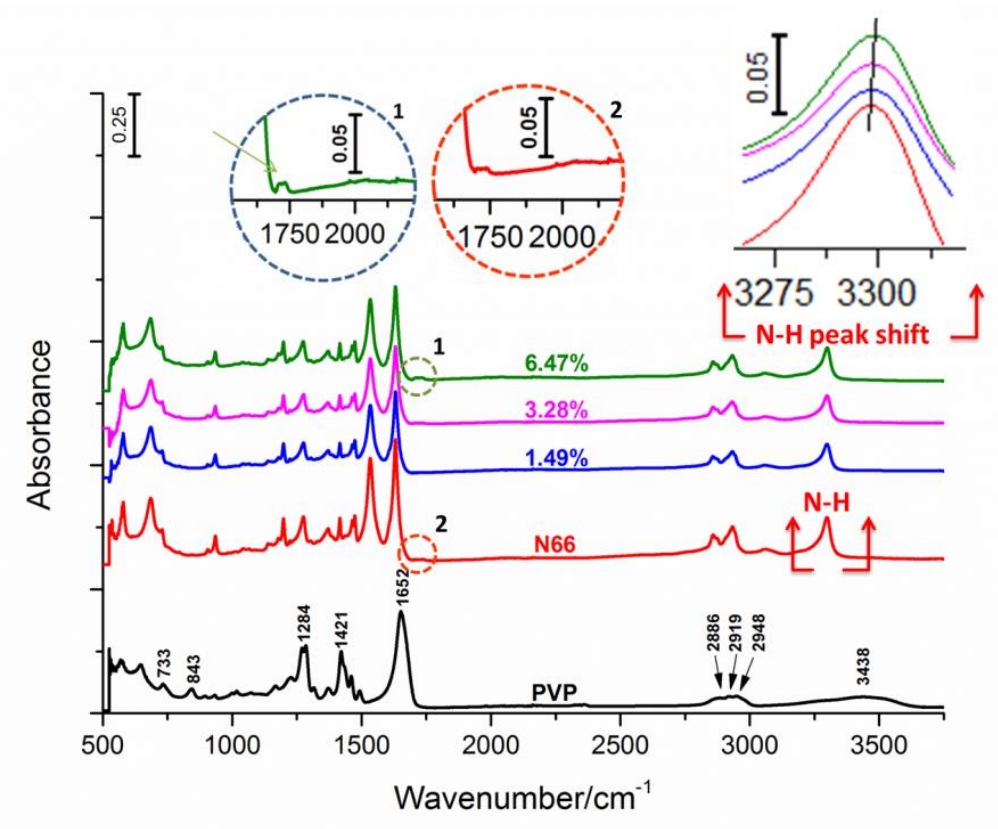

Figure 4.8: FTIR spectra of the PVP and the AgNW/N66 composite particles. 
Next, the spectra of AgNW/N66 composites are examined. We observe a systematic shift of the $\mathrm{N}-\mathrm{H}$ stretching peak to higher wavenumbers with increasing Ag content (i.e., from 0 to $6.47 \mathrm{wt} \%)$. We attribute this frequency shift to the cleavage of the hydrogen bonds between $\mathrm{N}-\mathrm{H}$ and $\mathrm{C}=\mathrm{O}$ in $\mathrm{N} 66$ as $\mathrm{AgNW}$ are introduced. As a result, the average stiffness of the $\mathrm{N}-\mathrm{H}$ bonds increases, and the $\mathrm{N}-\mathrm{H}$ peak exhibits higher energy shift.

We also find a peak intensity increase at $1710 \mathrm{~cm}^{-1}$ with an increased fraction of silver. It corresponds to the free $\mathrm{C}=\mathrm{O}$ end groups in N66 [61]. The free end groups are not associated with truly amide groups, thus they exhibit their unique $\mathrm{C}=\mathrm{O}$ stretching. We explain the intensity increase as follows. The AgNWs act as heterogeneous nucleating sites where polymer chains start to grow. With increased density of chains (per volume), the average chain length gets shorter and the density of chain-end-groups increases. Therefore, the $6.47 \mathrm{wt} \% \mathrm{Ag}$ composite has the highest density of end-groups (as well as shortest average chain length) and exhibits the highest intensity $1710 \mathrm{~cm}^{-1}$ peak.

\subsection{X-ray Diffraction}

XRD spectra of the AgNW/N66 composite particles were acquired as described in Section 3.5.3. The two strong reflections at $2 \theta=20.1^{\circ}$ and $24.0^{\circ}$ are the $(100)$ and $(010,110)$ planes of N66 $\alpha$ phase crystals [30], respectively (Fig. 4.9). The four peaks at $2 \theta=37.9^{\circ}, 44.0^{\circ}$, $64.2^{\circ}$ and $77.1^{\circ}$ conform to (111), (200), (220) and (311) planes of bulk silver. All the four reflections are assigned to face centered cubic (fcc) silver. In Fig. 4.9, all patterns are normalized by the intensity of the (100) peak. 
The mass ratios between $\mathrm{Ag}$ and N66 are proportional to their XRD intensity ratios. The XRD intensity of Ag was calculated by integrating the highest silver peak. The XRD intensity of N66 was calculated by integrating the two N66 peaks. This work was done using Jade 5. First, background and $\mathrm{CuK} \alpha 2$ scattering signal were removed by default setting of the software. Then, the curve was smoothened to reduce the noise. The Ag to N66 XRD intensity ratios were found as $7.92 \%, 13.89 \%$ and $28.50 \%$ in the order of increasing $\mathrm{Ag}$ content. These ratios are proportional to the mass ratios calculated in Chapter 3 as shown in the inset of Fig. 4.9.

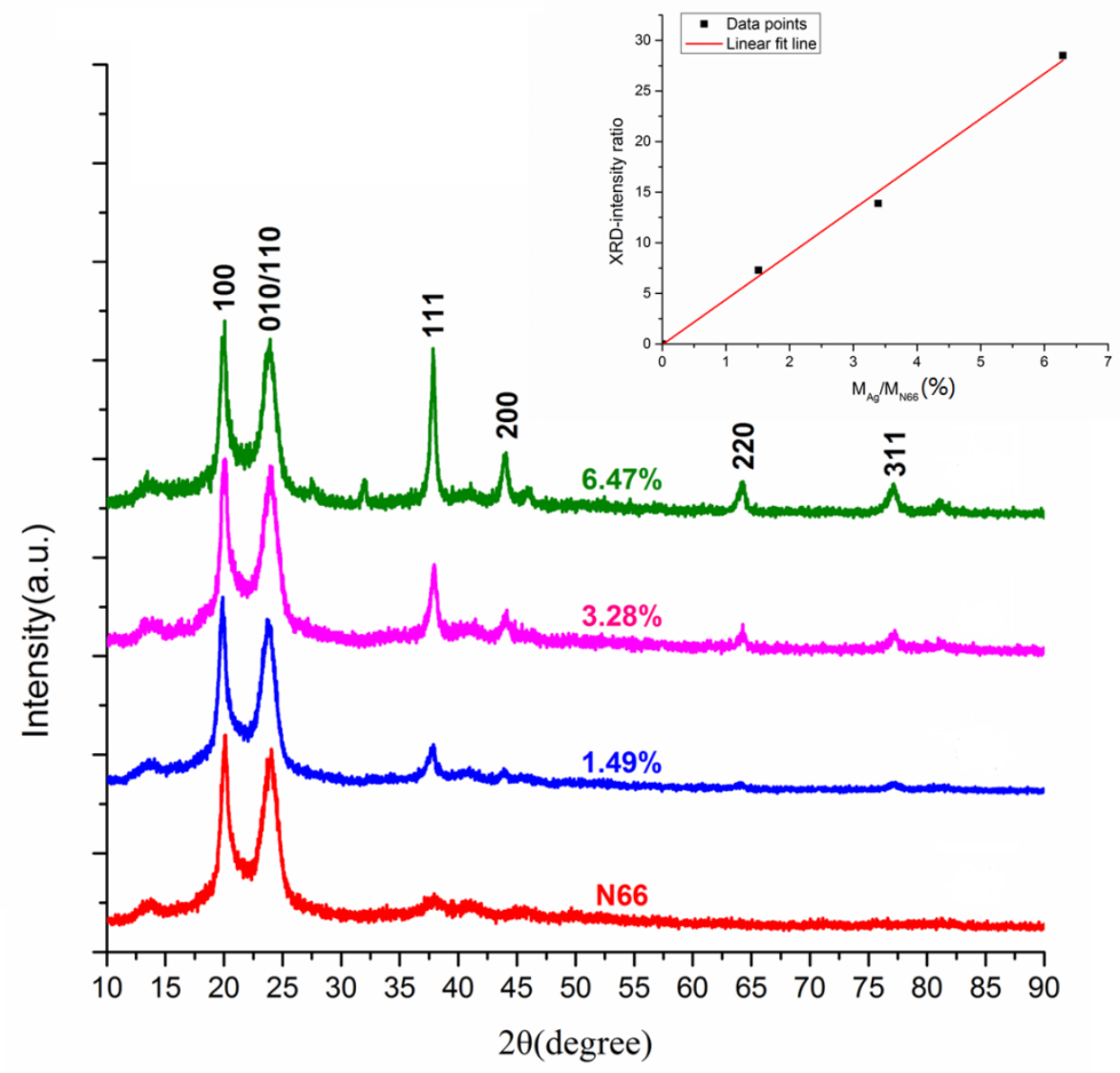

Figure 4.9: XRD spectra of AgNW/N66 composite particles. The inset shows AgNW:N66 mass ratio versus AgNW:N66 XRD intensity ratio. 


\subsection{Differential Scanning Calorimetry}

Melting point $\left(\mathrm{T}_{\mathrm{m}}\right)$, crystallization point $\left(\mathrm{T}_{\mathrm{c}}\right)$ and glass transition temperature $\left(\mathrm{T}_{\mathrm{g}}\right)$ of AgNW/N66 composites were derived from DSC measurements as described in Section 3.5.6. DSC thermographs of AgNW/N66 composites are shown in Fig. 4.10.

In Fig. 4.10a, the $\mathrm{T}_{\mathrm{g}}$ appears as a step in the baseline of the DSC signal. This is due to the sample undergoing a change in specific heat. DSC thermographs show that $T_{g}$ of AgNW/N66 composites decreases with increase of AgNW weight fraction. For example, $\mathrm{T}_{\mathrm{g}}$ drops from $61{ }^{\circ} \mathrm{C}$ to $48^{\circ} \mathrm{C}$ from control sample to $6.47 \mathrm{wt} \% \mathrm{Ag}$ composite sample.

We have two explanations for the reduction of $\mathrm{T}_{\mathrm{g}}$ with increase of $\mathrm{AgNW}$ weight fraction. First is the plasticizer effect. The AgNWs embed themselves between the chains of N66 polymers, and space the chains apart. This placement increases the free volume (empty internal space available for the movement of the polymer chains) (Fig. 4.11) in the polymer matrix and therefore increases the segmental mobility [62]. It is generally accepted that $\mathrm{T}_{\mathrm{g}}$ reduction for most polymers is proportional to the plasticizer concentration [63]. Second, as discussed in Section 4.3, the AgNWs act as heterogeneous nucleating sites and lead to shorter polymer chains. As a result, the segmental mobility of the chains increases accounting for lower $\mathrm{T}_{\mathrm{g}}$ [64]. Figure 4.12 shows the negative $\mathrm{T}_{\mathrm{g}}$ versus AgNW content. The curve exhibit super-linear behavior which indicates both plasticizer and shorter chain effects work at the same time. 

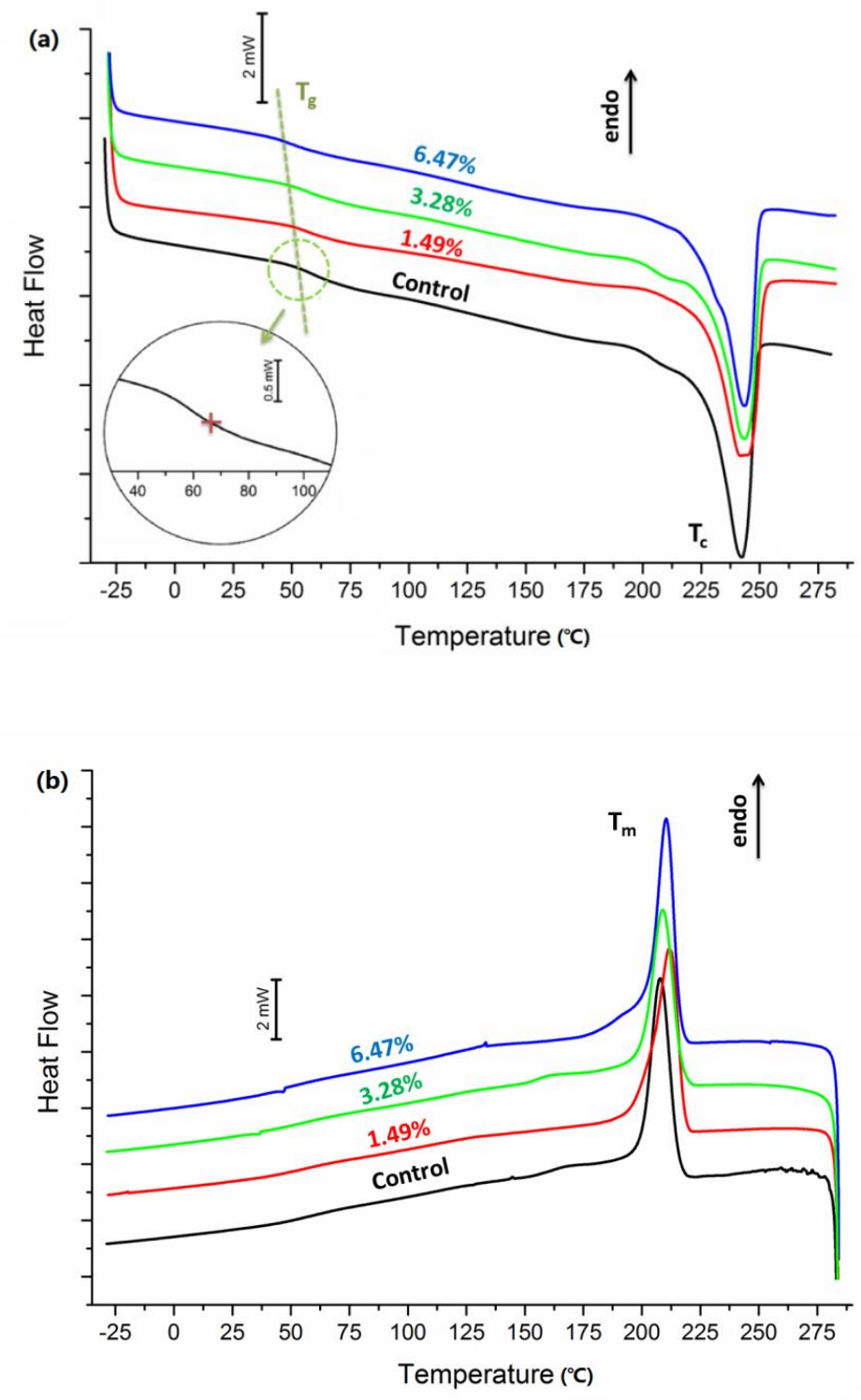

Figure 4.10: a) Crystallization diagram for AgNW/N66 composites. b) Melting diagram for AgNW/N66 composites. 


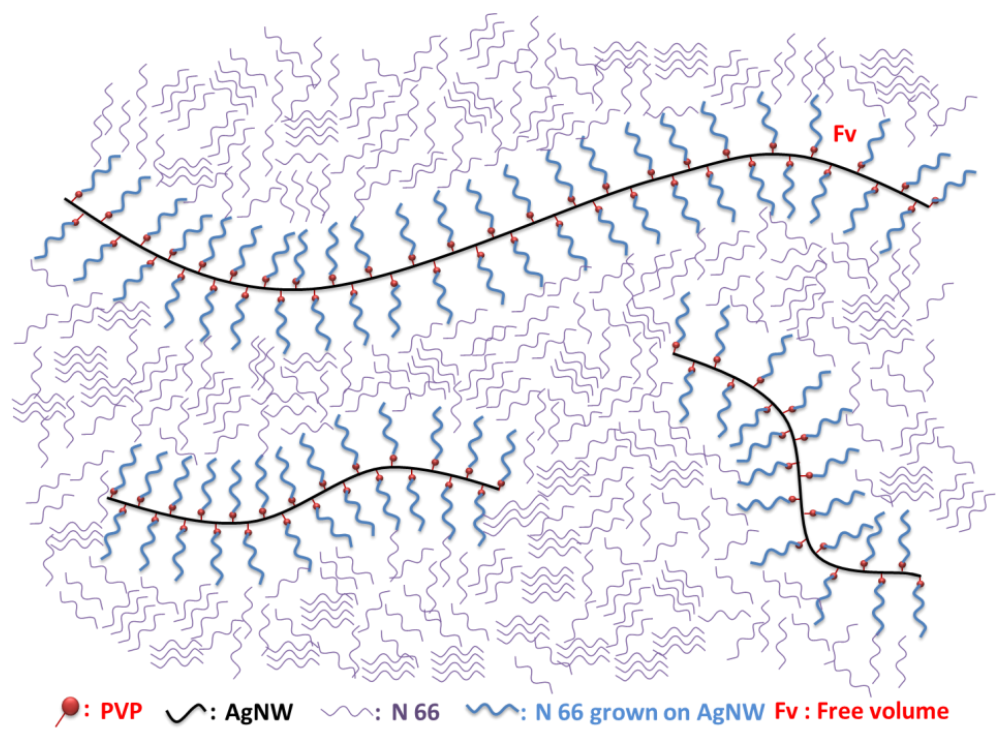

Figure 4.11: Schematic illustration of free volume formation in AgNW/N66 composite.

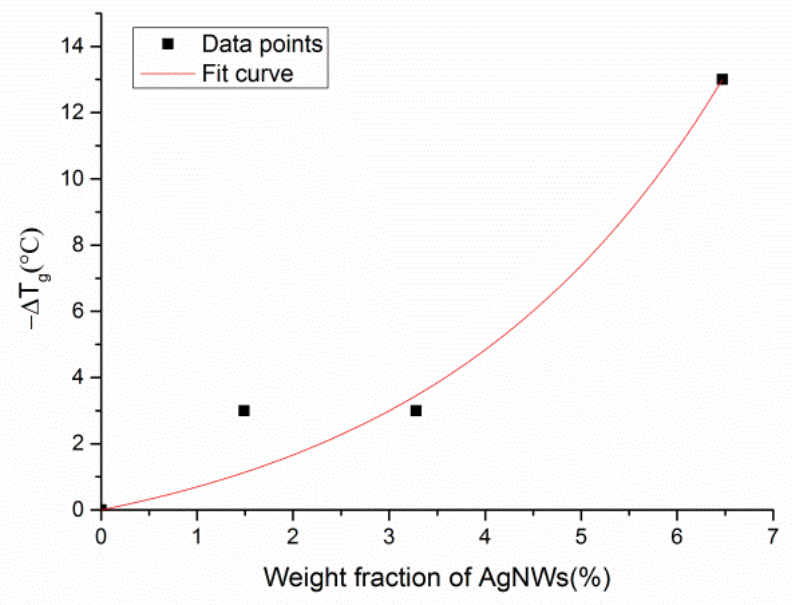

Figure 4.12: Negative $\Delta \mathrm{T}_{\mathrm{g}}$ versus $\mathrm{AgNW}$ content.

The melting/crystallization temperature of AgNW/N66 composites as deduced from Fig. 4.10 shows insignificant variation of $\mathrm{T}_{\mathrm{m}}$ and $\mathrm{T}_{\mathrm{c}}$ with silver content. The exothermic peaks of 
AgNW/N66 composites decrease from $242.1{ }^{\circ} \mathrm{C}$ to $241.5{ }^{\circ} \mathrm{C}$ from $6.47 \mathrm{wt} \%$ Ag composite to N66. The endothermic peaks of AgNW/N66 composites are between $208{ }^{\circ} \mathrm{C}$ and $211.1^{\circ} \mathrm{C}$ from $6.47 \mathrm{wt} \%$ Ag composite to N66. Silver nanowires act as heterogeneous nucleating sites. Generally, more nucleating sites will result in smaller crystals and higher crystallinity, thus lead to higher melting/crystallization temperature. However, in present work, we observe insignificant temperature variation. This situation could be due to the low amount of AgNWs in N66. The influence of AgNWs on the melting/crystallization behavior of the composites may be ignored.

\subsection{Mechanical properties by nano-indentation}

Mechanical properties of AgNW/N66 composites were investigated by nano-indentation as described in Section 3.5.7. Assuming a linearly elastic half-space and rigid conical indenter, Sneddon [65] found the load measured by the indenter can be expressed as $\mathrm{F}=\delta \mathrm{d}^{n}$, where $\delta$ and $\mathrm{n}$ are material constants and $\mathrm{d}$ is the displacement of the indenter. The load-displacement curves for AgNW/N66 composites are shown in Fig. 4.13. Then, using the load-displacement data, one can obtain the elastic modulus of the specimen using Eq. 4.1,

$$
E_{r}=\frac{S}{2} \sqrt{\frac{\pi}{A\left(\mathrm{~d}_{\mathrm{c}}\right)}}
$$

where $\mathrm{S}$ is the slope of the unloading curve in its initial regime, which is related to the combined modulus of indenter and material. $\mathrm{A}\left(\mathrm{d}_{\mathrm{c}}\right)$ is tip geometry. Oliver and Phar approach [66] shows $A\left(\mathrm{~d}_{\mathrm{c}}\right)=\eta \mathrm{d}_{\mathrm{c}}{ }^{2}$ where $\eta$ depends on indenter tip geometry [66]. The hardness $(\mathrm{H})$ is defined as effective maximum pressure imparted to the material during the indentation test. It is estimated from Eq. 4.2. 


$$
H=\frac{F_{\max }}{A\left(\mathrm{~d}_{\mathrm{c}}\right)}
$$

Modulus and hardness of AgNW/N66 composites are shown in Fig. 4.14. It can be seen both the modulus and hardness show insignificant variation with AgNW content. Halpin and Tsai [67] showed that the overall composite modulus $\left(\mathrm{E}_{\mathrm{c}}\right)$ could be expressed in terms of the corresponding matrix modulus $\left(\mathrm{E}_{\mathrm{m}}\right)$ and the reinforcing phase modulus $\left(\mathrm{E}_{\mathrm{f}}\right)$ as in Equation 4.3.

$$
\frac{E_{c}}{E_{m}}=\frac{1+\zeta \eta V_{f}}{1-\eta V_{f}}
$$

where $\eta$ is given by

$$
\eta=\frac{E_{f} / E_{m}-1}{E_{f} / E_{m}+\zeta}
$$

$\mathrm{V}_{\mathrm{f}}$ is the volume fraction of filler and $\zeta$ is the shape parameter, which is related to the filler geometry and loading direction.

Given constant matrix modulus, the composite modulus is approximately linearly proportional to the $\mathrm{V}_{\mathrm{f}}$ as predicted from Equation 4.3. However, as inferred from DSC and FTIR results, the matrix modulus decreases when filler content increases due to shorter polymer chains. The author hypothesizes the decreasing matrix modulus, $\mathrm{E}_{\mathrm{m}}$, obeys $\mathrm{E}_{\mathrm{m}}=\mathrm{E}_{\mathrm{mo}}\left(1-A V_{f}\right)$ where $\mathrm{E}_{\mathrm{mo}}$ is the matrix modulus without fillers. Thus, considering a sufficiently low value of $V_{f}$, he conjectures the insignificant variation of modulus and hardness with AgNW content is due to a counterbalance between filler enforcement and decrease in matrix modulus (Fig. 4.15). 


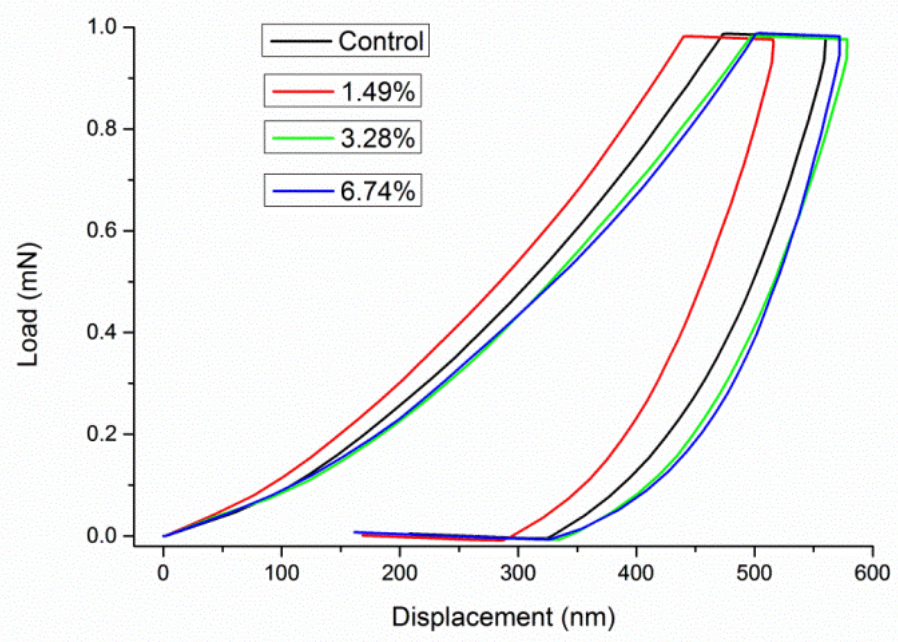

Figure 4.13: Load-displacement curve of AgNW/N66 composites.

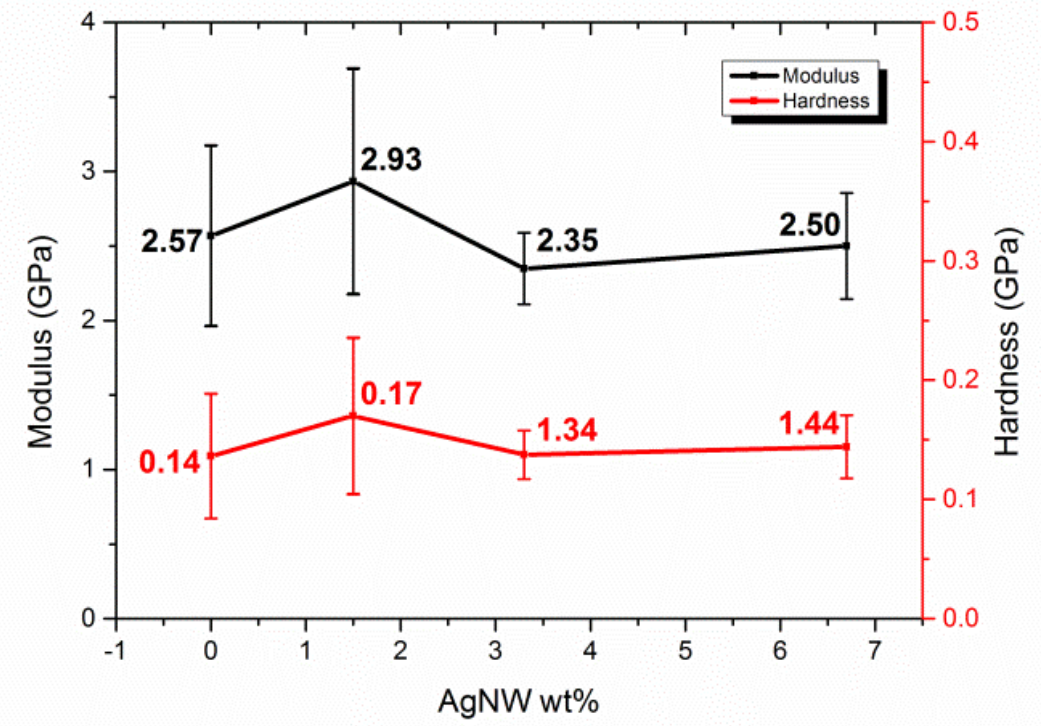

Figure 4.14: Modulus and hardness of AgNW/N66 composite samples. 


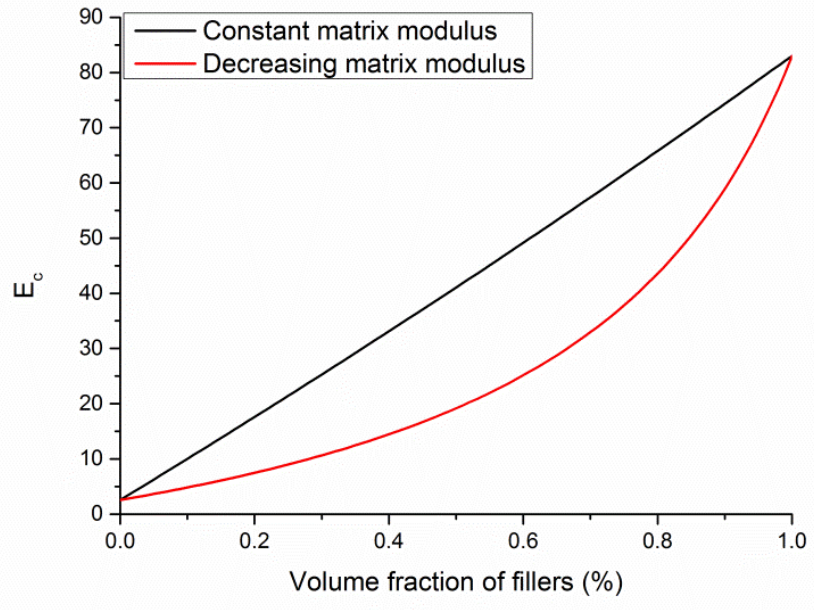

Figure 4.15: Composite modulus for constant versus decreasing matrix modulus with AgNW content. The model parameters are: $E_{f}=83 \mathrm{Gpa} ; E_{m o}=2.57 \mathrm{Gpa} ; \zeta=10 ; A=0.8$. 


\section{CHAPTER 5}

\section{Conclusions and Future Work}

\subsection{Conclusions}

In this thesis, a novel technique for manufacturing of polymer-based nanocomposites is developed, where dispersion of nanofillers in the polymer occurs simultaneously during polycondensation at room temperature. In particular, N66 composites with AgNW fillers are demonstrated. The following conclusions are drawn:

1) The mechanism of the simultaneous nanofiller dispersion and polymerization is understood to involve polycondensation in a nonpolar (but partially polar) solvent domain (e.g., THF). Present in this domain is at least one of the polymer precursors (monomer) as well as nanofillers. This nonpolar solvent domain has also boundaries with a polar one (e.g., water), which has dissolved at least one of the polymer precursors. During polymerization the precursor diffuses from the polar to nonpolar domain. Hence, the transport at the interface between nonpolar/polar solvent domain(s) rate limits and controls the polycondensation.

2) In the absence of mixing, polymer precursor transport occurs through a horizontal interface between the two solvents. It is found in the present thesis work that the rate of polymerization and dispersion can be dramatically accelerated by aggressive mixing by vortexing. Vortexing results in emulsification, where the nonpolar solvent segregates into microparticle domains in the polar solvent. Hence, polymerization occurs in the form of microparticles. The enhanced 
interface area between nonpolar/polar domains due to emulsification accelerates polymerization and nanofiller dispersion. Accordingly, the resultant composites are named as Nanocomposites by Dynamic Emulsification Polycondensation (NCDEP).

3) It is concluded that PVP plays multiple instrumental roles in DEP. First, it serves as a surfactant in efficient dispersion of AgNWs in water. Strong binding of PVP to Ag surface is verified by FTIR spectroscopy. Binding is by coordinative bonding through carbonyl moieties in PVP. Second, PVP also mediates the dispersion of AgNWs in the THF domains. Third, as elucidated by FTIR spectroscopy in this thesis, PVP establishes hydrogen bonding with N66. Hence, it functions as a coupling agent.

4) The $\mathrm{T}_{\mathrm{g}}$ of the $\mathrm{AgNW} / \mathrm{N} 66$ composites varies from 61 to $48^{\circ} \mathrm{C}$ as the weight content of $\mathrm{Ag}$ increases from 0 to $6.47 \%$. We explain this depression in $\mathrm{T}_{\mathrm{g}}$ in terms of two mechanisms. First, AgNWs act as plastizers and lead to increase in the free volume. However, by FTIR spectroscopy we show this plastizer effect can be more dramatic due to seeding effect of the AgNWs. It is observed that the IR signal characteristic of chain ends increase with $\mathrm{Ag}$ content, indicative of higher density of polymer chains. Hence, AgNWs serve as seeds, from which polymer chains nucleate. We anticipate these chains sticking out from the silver surface has a higher misalignment with the chains in the matrix leading to increased free volume. Second, the FTIR evidence of higher density but shorter chains corroborates another mechanism for the lowering of $\mathrm{T}_{\mathrm{g}}$. The shorter average chain length facilitates the slipping of polymer chains against each other. 
5) Dispersion of nanofillers in the N66 matrix is expected to yield enhanced mechanical stiffness and strength. However, our nano-indentation results show insignificant variation of Young's modulus and hardness with AgNW content. Based on Halpin-Tsai model, we conclude that the filler enforcement and shorter polymer chain effect counterbalance.

\subsection{Future work}

In the present thesis work, we were motivated towards mechanical reinforcement in N66 by dispersion of AgNWs. On the contrary, our investigation of the mechanical properties using nano-indentation did not reveal any reinforcement due to AgNWs. As discussed in the remainder of this thesis, the lack of mechanical reinforcement observed was attributed to the AgNW content being insignificantly low. Therefore, the future work should investigate AgNW/N66 composites of higher Ag content.

An interesting property of the AgNWs is their high electrical conductivity. Bulk silver has the highest metal conductivity among all metals. Thus, AgNW/N66 composites of the present thesis may be further investigated with the objective of developing transparent conductive layers. Transparent conductive layers based on AgNWs coated on glass substrates have already been demonstrated in the literature and they have been the subject of increasing interest due to their potential use in touch screens. However, those demonstrations lacked stabilization by a polymer.

It has been typically observed that the layer conductivity exhibits a sudden jump above a threshold AgNW density. Basically, the layer turns conductive when the AgNW density is high enough to assure percolation. Once percolation occurs, the nanowires form continuous conductive pathways. As a matter of fact, although it has not been investigated systematically, conductive 
AgNW/N66 composites were obtained during the course of the present thesis work when $\mathrm{Ag}$ weight content was increased to $26.8 \%$. Therefore, future work should investigate higher $\mathrm{Ag}$ content AgNW/N66 composites not only for mechanical reinforcement, but also for developing durable transparent conductive layers 


\section{REFERENCES}

[1] M. Biron, and O. Marichal. Thermoplastics and thermoplastic composites. Amsterdam: Elsevier, 2013.

[2] J. Jordan, K. I. Jacob, R. Tannenbaum, M. A. Sharaf, and I. Jasiuk, "Experimental trends in polymer nanocomposites-a review," Journal of Materials science \& Engineering A, vol. 393, pp. 1-11, 2005.

[3] B. Chen, W. Gao, J. Shen, and S.Guo, "The multilayered distribution of intumescent flame retardants and its influence on the fire and mechanical properties of polypropylene," Journal of Composites Science and Technology, vol. 93, pp. 54-60, 2014.

[4] Z. Zhang, B. Lin, N. Zhou, F. Yu and H. Zhang, "Flame-retardand olefin block copolymer composites with novel halogen-free intumescent flame retardants based on the composition of melamine phosphate and pentaerythritol," Journal of Applied Polymer Science, vol. 131, pp.40066 (1-10), 2014.

[5] S. Arunvisut, S. Phummanee, and A. Somwangthanaroj, "Effect of clay on mechanical and gas barrier properties of blown film LDPE/Clay nanocomposites," Journal of Applied Polymer Science, vol. 106, pp. 2210-2217, 2007.

[6] Y. Yang, L, Bolling, M. Priolo, and J, Grunlan, "Super gas barrier and selectivity of grapheme oxide-polymer multilayer thin films," Journal of Advanced materials, vol. 25, pp. 503-508, 2013.

[7] H. Perelshtein, G. Applerot, N. Perkas, G. Guibert, S. Mikhailov and A. Gedanken, "Sonochemical coating of silver nanoparticles on textile fabrics (nylon, polyster and cotton) and their antibacterial activity," vol. 19, pp. 1-6, 2008.

[8] M. T. Byrne and Y. K. Cun'ko, "Recent advances in research on carbon nanotubepolymer composites" Journal of Advance Materials, vol.22, pp.1672-1688, 2010.

[9] D. Nandi, S. Nandi, P. K. Pal, A. K. Ghosh, A. De and U.C.Ghosh, "Polypyrrole decorated grapheme nanostructure: Fabrication, depiction and anomalous dimensional crossover in electronic conduction" Journal of Applied Surface Science, vol. 293, pp. 9096, 2014.

[10] A. M. Marconnet, N. Yamamoto, M. A. Panzer, B. L. Wardle, and K.E. Goodson, "Thermal conduction in aligned carbon nanotube-polymer nanocomposites with high packing density," Journal of American Chemical Society, vol. 5, pp. 4818-4825, 2011.

[11] F. Hussain, M. Hojjati, M. Okamoto and R. E. Gorga, "Polymer-matrix nanocomposites, processing, manufacturing, and application: An overview," Journal of Composite 
Materials, vol. 40, pp. 1511-1575, 2006.

[12] S. Bhadrakumari and P. Predeep, "Elaboration and variable of polymer-impregnated superconduction ceramic composite," Journal of Superconductivity and Novel Magetism, vol. 23, pp. 233-236, 2010.

[13] R. Po, C. Carbonera, A. Bernardi, F.Tinti, and N. Camaioni, "Polymer-and carbon-based electrodes for polymer solar cells: toward low-cost, continuous fabrication over large area," Journal of Solar Energy Materials and Solar Cells, vol.100, pp. 97-114, 2012.

[14] G. Ma, J.L, K. Sun, H. Peng, J. Mu, and Z. Lei, " High performance solid-state supercapacitor with PVA-KOH-K3[Fe $(\mathrm{CN}) 6]$ gel polymer as electrolyte and separator," Journal of Power Sources, vol 256, pp. 281-287, 2014.

[15] M. Alexandre, and P. Dubois, "Polymer-layered silicate nanocomposites: preparation, properties and uses of a new class of materials," Journal of Materials Science and Engineering, vol. 28, pp. 1-63, 2000.

[16] P. W. Morgan and S. L. Kwolek, "Interfacial polycondensation. II. Fundamentals of polymer formation at liquid interfaces," vol. XL, pp. 299-327, 1959.

[17] M. L. Shiao, and S. V. Nair, "Effect of glass-fiber reinforcement and annealing on microstructure and mechanical behavior of nylon 6,6," Journal of Materials Science, vol. 29, pp. 1739-1752, 1994.

[18] A. Baji, Y. Mai, S. Wong, M. Abtahi, and X. Du, "Mechanical behavior of selfassembled carbon nanotube reinforced nylon 6,6 fibers," Journal of Composites Science and Technology, vol. 70, pp. 1401-1409, 2010.

[19] H, Zhang, C. Gao, Y. Wang, P. C.P. Watts, H. Kong, X. Cui, and D. Yan, “ In situ polymerization approach to multiwalled carbon nanotubes-reinforced nylon 1010 composites: Mechanical properties and crystallization behavior," Polymer Journal, vol. 47, pp. 113-122, 2006.

[20] M. Kang, S.J. Myung, and H. Jin, "Nylon 610 and carbon nanotube composite by in situ interfacial polymerization," vol. 47, pp. 3961-3966, 2006.

[21] M. Akay, D. F. O'Regan and R. S. Bailey, "Fracture toughness and impact behavior of glass-fibre-reinforced polymide 6,6 injection mouldings," vol. 55, pp. 109-118, 1995.

[22] X. Liu, Q. Wu, L.A. Berglund, J. Fan, and Z. Qi, "Polyamide 6-clay nanocomposites/polypropylene-grafted-maleic anhydride alloys," Polymer, vol. 42, pp. 8235-8239, 2001.

[23] W. Tang, X. Hu, J., Tang, and R. Jin, "Toughening and compatibilization of 
polyphenylene sulfide/nylon 66 blends with SEBS and maleic anhydride grafted SEBS triblock copolymers," Journal of Applied Polymer Science, vol. 106, pp. 2648-2655, 2007.

[24] J. Yang, Z. Zhang, and H. Zhang, "The essential work of fracture of polyamide 66 filled with TiO2 nanoparticles," Journal of Composites Science and Technology, vol. 65 pp. 2374-2379, 2005.

[25] H. Zhang, Z. Zhang, J. Yang, and K. Friedrich, "Temperature dependence of crack initiation fracture toughness of various nanoparticles filled polyamide 66," Polymer Journal, vol. 47, pp. 679-689, 2006.

[26] J. E. Mark, Physical properties of polymers handbook. LLC: Springer, 2007.

[27] H. F. Brinson and L. C. Brinson, Polymer engineering science and viscoelasticity. LLC: Springer, 2008.

[28] B. Mu, Q. Wang, H. Wang, and L. Jian, "Noisothermal crystallization kinetics of nylon 66/montmorillonite nanocomposites," Journal of Macromolecular Science, Part B: Physics. vol. 46(6), pp. 1093-1104, 2007.

[29] H. Wang, T. Shi, S. Yang, L. Zhai, and G. Hang, "Crystallization Behavior of PA66/SiO2 organic-inorganic hybrid material," Journal of Applied Polymer Science, vol, 101 pp. 810-817, 2006.

[30] Y. Lu, Y. Zhang, G. Zhang, M. Yang, S. Yan, and D. Dhen, "Influence of thermal processing on the perfection of crystals in polyamide 66 and polyamide 66/clay nanocomposites," Polymer Journal, vol. 45, pp. 8999-9009, 2004.

[31] H. Wang, B. Mu, J. Ren, L. Jian, J. Zhang, and S. Yang, "Mechanical and tribological behaviours of PA66/PVDF blends filled with calcium sulphate whiskers," Polymer Journal Composites, vol. 30, pp. 1326-1332, 2009.

[32] Z. Chen, X. Liu, T. Li, and R. Lü, "Mechanical and tribological properties of PA66/PPS blend. II. Filled with PTFE," Journal of Applied Polymer Science, vol. 101, pp. 969-977, 2006.

[33] Z. Chen, T. Li, Y. Yang, X. Liu, and R. Lv, "Mechanical and tribological properties of PA/PPS blends," Journal of Wear, vol. 257, pp. 696-707, 2004.

[34] Z. Chen, T. Li, X. Liu and R. Lü, "Friction and wear mechanisms of polyamide 66/high density polyethylene blends," Polymer Journal Science: Part B: Polymer Physics, vol. 43, pp. 2514-2523, 2005.

[35] G. Zhuang, Y. Gui, Y. Yang, B. Li and J. Zhang, "Tremolite-reinforced nylon 66 
composites: Mechanical and rheological properties," Journal of Applied Polymer Science, vol. 69, pp. 589-598, 1998.

[36] Z. Zhang, J. Yang and K. Friedrich, "Creep resistant polymeric nanocomposites," Polymer Journal, vol.45, pp. 3481-3485, 2004.

[37] B Ribeiro, LB Nohara, SS Oishi, ML Costa and EC Botelho, "Nonoxidative thermal degradation kinetic of polyamide 6,6 reinforced with carbon nanotubes," Journal of Thermoplastic Composite materials, vol. 26(10), pp. 1317-1331, 2012.

[38] M. Tarameshlou, S. H. Jafari, H. A. Khonakdar, M. Farmahini-Farahani and S. Ahmadian, "Synthesis of exfoliated polyamide 6,6/organically modified montmorillonite nanocomposites by in situ interfacial polymerization," Polymer Journal Composites, vol. 28, pp. 733-738, 2007.

[39] S. Zeng, K. Yong, I. Roy, X. Dinh, X. Yu and F. Luan, “A review on functionalized gold nanoparticles for biosensing applications," Journal of Plasmonics, vol. 6, pp. 491-506, 2011.

[40] S. Karumuri, "Hybrid surface plasmon damping chemical sensor." vol. Master: Oklahoma State University, 2009.

[41] Rama K. R.G. Ede, "Plasmon-damping chemical sensor for hydrogen fuel monitoring." vol. Master: Oklahoma State University, 2011.

[42] K. L. Kelly, E. Coronade, L. L. Zhao, and G. C. Schatz, "The optical properties of metal nanoparticles: The influence of size, shape, and dielectric environment," Journal of Physical chemistry, vol. 17, pp.668-677, 2003.

[43] A. A. Ashkarran, and A. Bayat, "Surface plasmon resonance of metal nanostructures as a complementary technique for microscopic size measurement," Journal of International Nano Letters, vol. 3, pp. 50-60, 2013.

[44] J. P. Kottmann, "Numerical Study of Plasmon Resonant Nanowires." Vol. Sc.D,: Swiss Federal Institute of Technology, 2001.

[45] G. Schider, J. R. Krenn, A. Hohenau, H. Ditlbacher, A. Leitner, F. R. Aussenegg, W. L. Schaich, I. Puscasu, B. Monacelli, and G. Boreman, "Plasmon dispersion relation of Au and Ag nanowires," Physical Review B. vol. 68(15), pp. 1-4, 2003.

[46] M. Song, G. Chen, Y. Liu, E. Wu, B. W, and H. Zeng, "Polarization properties of surface plasmon enhanced photoluminescence from a single Ag nanowire," Optics express vol. 20, pp. 22290-22297, 2012.

[47] H. Mao, J. Feng, X. Ma, C. Wu, and X. Zhao, "One-dimensional silver nanowires 
synthesized by self-seeding polyol process," Journal of nanoparticle research, vol. 14, pp. 887-902, 2012.

[48] Y. Gao, P. Jiang, L. Song, L, Liu, X. Yan, Z. Zhou, D. Liu, J. Wang, H. Yuan, Z. Zhang, X. Zhao, X. Dou, W. Zhou, G. Wang and S. Xie, "Growth mechanism of silver nanowires synthesized by polyvinylpyrrolidone-assisted polyol reduction," Journal of Physics D: Applied Physics, vol. 38, pp. 1061-1067, 2005.

[49] Y. Sun, and Y. Xia, "Large-scale synthesis of uniform silver nanowires through a soft, self-seeding, polyol process," Journal of Advanced Materials, vol. 14, pp. 833-837, 2002.

[50] Y. Sun, Y. Yin, B. T. Mayers, T. Herricks, and Y. Xia, "Uniform silver nanowires synthesis by reducing AgNO3 with ethylene glycol I the presence of seeds and poly(vinyl pyrrolidone)," Chemistry of Materials, vol. 14, pp. 4736-4745, 2002.

[51] Y. Sun, B. Gates, B. Mayers, and Y. Xia, "Crystalline silver nanowires by soft solution processing," Nano Letters, vol. 2, pp. 165-168, 2002.

[52] C. Chen, L. Wang, G. Jiang, Q. Yang, J. Wang, H. Yu, T. Chen, C. Wang and X. Chen, "The influence of seeding conditions and shielding gas atmosphere on the synthesis of silver nanowires through the polyol process," Journal of Nanotechnology, vol. 17, pp. 466-474, 2006.

[53] S. Coskun, B. Aksoy, and H. E. Unalan, "Polyol synthesis of silver nanowires: An extensive parametric study," Journal of Crystal Growth\&Design, vol. 11, pp. 4963-4969, 2011.

[54] B. J. Wiley, S. H. Im, Z. Li, J. McLellan, A. Siekkinen, and Y. Xia, "Maneuvering the surface plasmon resonance of silver nanostructures through shape-controlled synthesis," Journal of Physical Chemistry B, vol. 110, pp. 15666-15675.

[55] K. L. Kelly, E. Coronade, L. Zhao, and G. C. Schatz, "The optical properties of metal nanoparticles: The influence of size, shape, and dielectric environment," Journal of J Physical Chemistry B, vol. 107, pp. 668-677, 2003.

[56] K. Sivaiah, B. H. Rudramadevi, and S. Buddhudu, "Structural, thermal and optical properties of $\mathrm{Cu}^{2+}$ and $\mathrm{Co}^{2+}$ : PVP polymer films. Indian Journal of Pure \& Applied Physics," vol. 48, pp. 658-662, 2010.

[57] Y. Borodko, S. E. Habas, M. Koebel, P. Yang, H. Frei, and G. A. Somorjai, "Probing the interaction of poly(vinylpyrrolidone) with platinum nanocrystals by UV-Raman and FTIR,” Journal of Physical Chemistry B, vol. 110, pp. 23052-23059, 2006.

[58] Y. Du, and S. M. George, "Molecular Layer Deposition of Nylon 66 Films Examined Using in Situ FTIR Spectroscopy," Journal of Physical Chemistry C, vol. 111, pp. 8509- 
8517, 2007.

[59] N. Vasanthan, "Crystallinity Determination of Nylon 66 by Density measurement and fourier transform infrared (FTIR) spectroscopy," vol. 89, pp. 387-390, 2012.

[60] N. Giri, R. K. Natarajan, S. Gunasekaran, and S.Shreemathi, "13C NMR and FTIR spectroscopic study of blend behavior of PVP and nano silver particles," Archives of Applied Science Research, vol. 3(5), pp. 624-630, 2011.

[61] J. M. de Gooijer, J. Ellmann, M. Moller, and C. E. Koning, "End group modification of polyamide-6 in supercritical and subcritical fluids Part 3: Amine end group modification with diketene and diketene acetone adduct in $\mathrm{CO}_{2}$," Journal of Supercritical Fluids, vol. 31, pp. 75-87, 2004.

[62] J. Rudnick, and P. L. Taylor, "Theory of free volume in polymers," vol. 17, pp. 311-320, 1979.

[63] Z. Dong, "A study in glassy transition of pure and plasticized polymers including polymer plasticized by pressurized gas" vol. Ph.D.: University of Cincinnati, 1996.

[64] Y. Miwa, T. Tanase, K. Yamamoto, M. Sakaguchi, M. Sakai, and S. Shimade, "Influence of chain end and molecular weight on molecular motion of polystyrene, revealed by the ESR selective spin-label method," vol. 36, pp. 3235-3239, 2003.

[65] I. N. Sneddon, "Boussinesq's problem for a rigid cone," Proc. Cambridge Philos. Soc., vol. 44, pp. 492-507, 1948.

[66] W. C. Oliver, and G. M. Pharr, "An improved technique for determining hardness and elastic modulus using load and displacement sensing indentation experiments," Journal of Materials Research, vol. 7, pp. 1564-1582, 1992.

[67] J. C. Halpin, "Primer on composite materials," Journal of Materials \& Design, vol. 6, pp. 145-145, 1984. 
VITA

Linqi Zhang

Candidate for the Degree of

Master of Science

Thesis: Ag-NYLON NANOCOMPOSITES BY DYNAMIC EMULSION

POLYCONDENSATION

Major Field: Mechanical Engineering

Biographical:

Education:

Completed the requirements for the Master of Science in Mechanical Engineering at Oklahoma State University, Stillwater, Oklahoma in December, 2014.

Completed the requirements for the Bachelor of Science in Mechanical Engineering at Harbin Institute of Technology, Harbin, Heilongjiang, China in June, 2012.

Experience:

Graduate Research Assistant, MAE, OSU Stillwater (Jan. 2013 - Aug. 2013 )

Graduate Teaching Assistant, MAE, OSU Stillwater (Aug. 2013 - May. 2014)

Graduate Teaching Assistant, MAE, OSU Stillwater (Aug. 2013 - Dec. 2014) 